\title{
Two-loop operator matrix elements calculated up to finite terms for polarized deep inelastic lepton-hadron scattering
}

\author{
Y. Matiounine, ${ }^{1}$ J. Smith, ${ }^{1}$ and W. L. van Neerven ${ }^{2}$ \\ ${ }^{1}$ Institute for Theoretical Physics, State University of New York at Stony Brook, Stony Brook, New York 11794-3840 \\ ${ }^{2}$ Instituut-Lorentz, University of Leiden, P.O. Box 9506, 2300 RA Leiden, The Netherlands
}

(Received 27 March 1998; published 4 September 1998)

\begin{abstract}
We present the two-loop corrected operator matrix elements contributing to the scale evolution of the longitudinal spin structure function $g_{1}\left(x, Q^{2}\right)$ calculated up to finite terms which survive in the limit $\varepsilon=N$ $-4 \rightarrow 0$. These terms are needed to renormalize the local operators up to third order in the strong coupling constant $\alpha_{s}$. Further the expressions for the two-loop corrected operator matrix elements can be inserted into one loop graphs to obtain a part of the third order contributions to these matrix elements. This work is a first step in obtaining the third order anomalous dimensions so that a complete next-to-next-to-leading order analysis of the above-mentioned structure function can be carried out. In our calculation particular attention is paid to the renormalization constant which is needed to restore the Ward identities violated by the HVBM prescription for the $\gamma_{5}$ matrix in $N$-dimensional regularization. [S0556-2821(98)04419-1]

PACS number(s): 11.15.Bt, 12.38.Bx
\end{abstract}

\section{INTRODUCTION}

During the last ten years there has been a lot of activity in the area of spin physics in particular the study of the structure functions $g_{i}\left(x, Q^{2}\right) \quad(i=1,2)$ measured in polarized lepton-hadron scattering (for reviews see Refs. [1] and [2]). One of the achievements was the determination of the anomalous dimensions of twist two local composite operators up to two-loop order (see Refs. $[3,4]$ ) which determine the scale $\left(Q^{2}\right)$ evolution of the longitudinal spin structure function $g_{1}\left(x, Q^{2}\right)$.

Since also the order $\alpha_{s}$ contributions to the coefficient functions are known (see Refs. [5,6]) it is now possible to make a full next-to-leading order (NLO) analysis of $g_{1}\left(x, Q^{2}\right)$ analogous to what has been done for the spin averaged structure function $F_{2}\left(x, Q^{2}\right)$. When the statistics of the ongoing experiments improve it will be also necessary to investigate how the NLO predictions are modified by including yet higher order corrections. In particular this will be interesting for the study of the small $x$ region where gluon contributions to the anomalous dimensions and the coefficient functions are important. A start of this program, which will lead to a complete next-to-next-to-leading order (NNLO) description of the longitudinal spin structure function, has been made in Ref. [7] where quark as well as gluon coefficient functions have been computed up to order $\alpha_{s}^{2}$. The three-loop anomalous dimensions are still missing.

In this paper we want to make the first step in a three loop program by computing the operator matrix elements (OME's), obtained by sandwiching the local composite operators between quark and gluon states, up to two-loop order including nonpole terms which are finite in the limit $N \rightarrow 4$. Here $N$ refers to the method of $N$-dimensional regularization which is used to regularize the ultraviolet divergences occurring in the OME's.

Using this method the latter divergences manifest themselves as pole terms of the type $1 /(N-4)^{k}$. These finite terms are needed to renormalize the three-loop graphs contributing to the OME's. Further one can use the unrenormalized two-loop expressions to determine a part of the threeloop contributions to the OME's by inserting them into one loop diagrams. Another interesting feature of the calculation of the spin OME's is the appearance of the $\gamma_{5}$ matrix and the Levi-Civita tensor in the operator vertices. Since we use $N$-dimensional regularization one has to find a suitable prescription to extend these objects to $N$ dimensions. In this work we have chosen the HVBM prescription given first by 't Hooft and Veltman [8] and worked out in more detail by Breitenlohner and Maison [9]. One of the characteristics of this prescription is that the $\gamma_{5}$ matrix commutes with the other $\gamma_{\mu}$ matrices for $N>4$.

Although this is a consistent scheme, since it preserves the cyclicity of the traces, it violates some Ward identities which would be preserved with an anticommuting $\gamma_{5}$ matrix. To restore these Ward identities one has to introduce additional renormalization factors called $Z_{q q}^{5, r}$. These factors will be calculated up to order $\alpha_{s}^{2}$ for the nonsinglet ( $r=\mathrm{NS}$ ) and singlet $(r=\mathrm{S})$ operators for arbitrary spin.

The paper is organized as follows. In Sec. II we present the algebraic formulas for the unrenormalized OME's expressed in renormalization group coefficients. We determine the factors $Z_{q q}^{5, r}$ for $r=\mathrm{NS}$ (nonsinglet) and S (singlet) and compare our results with earlier calculations in the literature. The long expressions for the spin OME's, which are the results of our calculations, are given in Appendix A.

\section{THE CALCULATION OF THE TWO-LOOP OPERATOR MATRIX ELEMENTS}

In this section we will give an outline of the calculation of the OME's up to two-loop order. The operators, which appear in polarized lepton-hadron scattering, can be split into singlet and nonsinglet parts with respect to the flavor group. In leading twist (namely, 2) the nonsinglet quark operator of spin $n$ is given by

$$
\begin{aligned}
O_{q, k}^{5, \mu_{1}, \mu_{2} \cdots \mu_{n}}= & \frac{1}{2} i^{n-1} \mathcal{S}\left[\bar{\psi}(x) \gamma_{5} \gamma^{\mu_{1}} D^{\mu_{2}} \ldots D^{\mu_{n}} \frac{\lambda_{k}}{2} \psi(x)\right. \\
& + \text { trace terms }] .
\end{aligned}
$$


In the singlet case there are two operators. The quark operator is represented by

$$
\begin{aligned}
O_{q}^{5, \mu_{1}, \mu_{2} \cdots \mu_{n}}= & \frac{1}{2} i^{n-1} \mathcal{S}\left[\bar{\psi}(x) \gamma_{5} \gamma^{\mu_{1}} D^{\mu_{2}} \cdots D^{\mu_{n}} \psi(x)\right. \\
& + \text { trace terms }]
\end{aligned}
$$

and the gluon operator is given by

$$
\begin{aligned}
O_{g}^{5, \mu_{1}, \mu_{2} \cdots \mu_{n}}= & \frac{1}{2} i^{n-2} \\
& \times \mathcal{S}\left[\epsilon^{\mu_{1} \alpha \beta \gamma} F_{\beta \gamma}(x) D^{a, \mu_{2}} \ldots D^{\mu_{n-1}} F_{\alpha}^{a, \mu_{n}}(x)\right. \\
& + \text { trace terms }] .
\end{aligned}
$$

In the composite operators above $\psi$ and $F_{\mu \nu}^{a}$ stand for the quark field and the gluon field tensor respectively. The $\lambda_{k}$ in Eq. (2.1) represent the generators of the flavor group and the index $a$ in Eq. (2.3) stands for the color. Further the above operators are irreducible tensors with respect to the Lorentz group so that they have to be symmetric and traceless in all their Lorentz indices $\mu_{i}$. From the operators above one can derive the Feynman rules for the operator vertices in the standard way (see, e.g., Refs. [3] and [11]). This derivation is facilitated if the operators are multiplied by the source

$$
J_{\mu_{1} \mu_{2} \cdots \mu_{n}}=\Delta_{\mu_{1}} \Delta_{\mu_{2}} \cdots \Delta_{\mu_{n}},
$$

with $\Delta^{2}=0$ in order to eliminate the trace terms in Eqs. (2.1)-(2.3). Hence all operator vertices in momentum space are multiplied by a factor $(\Delta \cdot p)^{n}$. For the computation of the OME's denoted by

$$
A_{i j}^{5}=\left\langle j(p)\left|O_{i}^{5}\right| j(p)\right\rangle
$$

with $i, j=q, g$ we choose the Feynman gauge except for the one-loop graphs for which we take the general covariant gauge. For this choice the gluon propagator equals

$$
\Delta_{a b}^{\mu \nu}(k)=\frac{i \delta_{a b}}{k^{2}}\left(-g^{\mu \nu}+(1-\xi) \frac{k^{\mu} k^{\nu}}{k^{2}}\right) .
$$

The matrix element (2.5) has to be considered as a connected Green function with the external legs amputated but with the external self energies of the partons $j$ included. In this paper all quarks and gluons are taken to be massless and the external momentum $p$ is off-shell $\left(p^{2}<0\right)$ in order to get finite expressions for the OME's. This choice implies that the OME's are not gauge invariant so that they cease to be ordinary $S$-matrix elements. Therefore they acquire unphysical parts which usually vanish by virtue of the equations of motion $(\mathrm{EOM})$ if the external legs are on shell. Contrary to the spin averaged operators treated, e.g., in Ref. [12] there is no mixing between gauge invariant (GI) or physical (phys) operators and nongauge invariant (NGI) operators (see Refs. [10-18]). Therefore the renormalization of the operators in Eqs. (2.1)-(2.3) is much easier than is the case for the spin averaged operators in Ref. [12]. On the other hand, due to the presence of the $\gamma_{5}$ matrix and the Levi-Civita tensors in the composite operators above, one has to correct for spurious terms in the OME's if one adopts $\mathrm{N}$-dimensional regularization.

The calculation of the Feynman graphs corresponding to the physical operators in Eqs. (2.1)-(2.3), which are depicted in the figures in Ref. [3], proceeds in the standard way. The corresponding Feynman integrals reveal ultraviolet divergences which are regularized using the method of $N$-dimensional regularization. In this way the above divergences show up in the form of pole terms of the type $(1 / \varepsilon)^{k}$ with $\varepsilon=N-4$. In Refs. [3,4] it was sufficient to evaluate the one-loop graphs up to finite and the two-loop graphs up to single pole terms in order to get the second order anomalous dimensions. Here we have to include terms proportional to $\varepsilon$ in the one-loop expressions and the two-loop graphs have to be computed up to finite terms. The way to compute the two-loop Feynman integrals up to finite terms is presented in Refs. [12,16], and Appendix B of Ref. [19]. We used the program FORM [20] to do the necessary algebra.

As has been mentioned above, the presence of the $\gamma_{5}$ matrix, which is essentially a four-dimensional object, will cause some technical problems when one chooses the method of $N$-dimensional regularization.

One has to find a prescription to give a suitable definition valid for all space-time dimensions.

We will adopt the HVBM prescription given by 't Hooft and Veltman [8] which has been worked out in more detail by Breitenlohner and Maison [9]. In order to facilitate the calculation of the OME's it is more convenient (see Refs. $[21,22])$ to replace the term $\Delta \gamma_{5}$ appearing in the operator vertices (see Appendix A in Ref. [3]) by

$$
\Delta \gamma_{5}=\frac{i}{6} \epsilon_{\mu \rho \sigma \tau} \Delta^{\mu} \gamma^{\rho} \gamma^{\sigma} \gamma^{\tau}
$$

Notice that this replacement is only equivalent to the HVBM prescription if a single $\gamma_{5}$ matrix is present in the numerator of the OME's, which is the case here. Thus only one LeviCivita tensor appears in all the numerators of the Feynman integrals so that the $\gamma_{5}$ matrix and the Levi-Civita tensor are on an equal footing. Since one has to be careful with the treatment of the Levi-Civita tensor, which is also a fourdimensional object, we have to follow the procedure in Appendix B of Ref. [3]. First one computes the numerator in the Feynman integral corresponding to a specific graph.

This numerator contains the integration momenta, which are in $\mathrm{N}$-spacetime dimensions. Then one applies tensorial reduction to express the whole integral into tensors containing $\Delta_{\mu}$ in Eq. (2.4) and $p_{\mu}$ in Eq. (2.5), which are $N$-dimensional vectors. Finally one has to project the whole matrix element on the tensor structure characteristic of the specific OME under consideration, which follows from Lorentz covariance in four dimensions. Therefore the Lorentz indices of $p_{\mu}$ and $\Delta_{\mu}$ with $N>4$ are simply dropped.

The advantage of the HVBM prescription is that the cyclicity of the traces is preserved. On the other hand it destroys the anticommutativity of the $\gamma_{5}$ matrix. This will lead to a renormalization of the nonsinglet axial-vector current 
$O_{q, k}^{5, \mu}$ in Eq. (2.1) in spite of the fact that it is conserved [23]. Furthermore as was shown in Ref. [22] the Adler-Bardeen theorem [24] is no longer true. The anticommutativity and the Ward identities can be restored by introducing an additional renormalization constant $Z_{q q}^{5, r}(r=\mathrm{NS}, \mathrm{S})$ which, when expressed in unrenormalized quantities, has the following form:

$$
\begin{aligned}
\hat{Z}_{q q}^{5, r}=1 & +\hat{a}_{s} S_{\varepsilon}\left[z_{q q}^{(1)}+\varepsilon z_{q q}^{\varepsilon,(1)}\right]+\hat{a}_{s}^{2} S_{\varepsilon}^{2} \\
& \times\left[-\frac{1}{\varepsilon} \beta_{0} z_{q q}^{(1)}+z_{q q}^{r,(2)}-2 \beta_{0} z_{q q}^{\varepsilon,(1)}-\hat{\xi} \frac{d z_{q q}^{\varepsilon,(1)}}{d \hat{\xi}} z_{\xi}\right]_{\hat{\xi}=1} .
\end{aligned}
$$

The hat indicates that all quantities are unrenormalized with respect to coupling constant $\alpha_{s}$, gauge constant $\xi$, and, in the case of the OME's, also the operator renormalization. Here $S_{\varepsilon}$ denotes the spherical factor characteristic of $N$-dimensional regularization

$$
S_{\varepsilon}=\exp \left[\frac{\varepsilon}{2}\left(\gamma_{E}-\ln 4 \pi\right)\right]
$$

where $\gamma_{E}$ denotes the Euler constant. Further we introduce a shorthand notation for the strong coupling constant so that

$$
a_{s}=\frac{\alpha_{s}}{4 \pi}, \quad \alpha_{s}=\frac{g^{2}}{4 \pi} .
$$

If we apply coupling constant renormalization

$$
\hat{a}_{s}=a_{s}\left[1+a_{s} S_{\varepsilon}\left(2 \beta_{0} \frac{1}{\varepsilon}\right)\right]
$$

and gauge constant renormalization

$$
\hat{\xi}=\xi\left[1+a_{s} S_{\varepsilon}\left(z_{\xi} \frac{1}{\varepsilon}\right)\right]
$$

where both are presented in the $\overline{\mathrm{MS}}$ scheme, we obtain

$$
Z_{q q}^{5, r}=1+a_{s} S_{\varepsilon}\left[z_{q q}^{(1)}+\varepsilon z_{q q}^{\varepsilon,(1)}\right]+a_{s}^{2} S_{\varepsilon}^{2}\left[\frac{1}{\varepsilon} \beta_{0} z_{q q}^{(1)}+z_{q q}^{r,(2)}\right] .
$$

In QCD $[\mathrm{SU}(N)]$ the renormalization group coefficients are given by

$$
\beta_{0}=\frac{11}{3} C_{A}-\frac{4}{3} n_{f} T_{f}, \quad z_{\xi}=C_{A}\left(-\frac{10}{3}-(1-\xi)\right)+\frac{8}{3} n_{f} T_{f},
$$

with $C_{F}=\left(N^{2}-1\right) / 2 N, C_{A}=N, T_{f}=1 / 2$, and $n_{f}$ stands for the number of light flavors. Notice that the coefficients $z_{q q}^{(1)}$ and $z_{q q}^{r,(2)}$ in Eqs. (2.8) and (2.13) are gauge ( $\xi$ ) independent in contrast to the terms proportional to $\varepsilon$ such as $z_{q q}^{\varepsilon,(1)}$ which depend on the gauge. As we will show later on $z_{q q}^{(1)}$ is uni- versal but $z_{q q}^{\varepsilon,(1)}$ and $z_{q q}^{r,(2)}$ depend on the given quantity. In the nonsinglet case $Z_{q q}^{5 \text {,NS }}$ can be inferred from the ratio

$$
Z_{q q}^{5, \mathrm{NS}}=\frac{\hat{A}_{q q}^{\mathrm{NS}, \text { phys }}}{\hat{A}_{q q}^{5, \mathrm{NS}, \mathrm{phys}}}
$$

where $\hat{A}_{q q}^{\mathrm{NS}, \text { phys }}$ and $\hat{A}_{q q}^{5 \text {,NS,phys }}$ denote the physical parts (for definitions see below) of the OME's without and with the $\gamma_{5}$ matrix, respectively. The former OME has been fully calculated in Ref. [12] whereas the latter OME will be presented below and in Appendix A. Notice that the numerator and the denominator would be identical if we applied the so-called naive (or anticommuting) $\gamma_{5}$ prescription. The latter method allows us to anticommute this matrix with the other $\gamma_{\mu}$ matrices so that it reaches another vertex in the diagram and leads to $Z_{q q}^{5, \mathrm{NS}}=1$. Therefore the renormalization constant $Z_{q q}^{5, \mathrm{NS}}$ restores the anticommutativity of the $\gamma_{5}$ matrix. The singlet coefficient which can be split into

$$
z_{q q}^{\mathrm{S},(2)}=z_{q q}^{\mathrm{NS},(2)}+z_{q q}^{\mathrm{PS},(2)}
$$

can also be calculated by anticommuting the $\gamma_{5}$ matrix.

The computation of the purely singlet part $z_{q q}^{\mathrm{PS},(2)}$ will be presented at the end of this section.

To check our results for the sums of the Feynman graphs it is useful to have explicit expressions for the pole terms. Therefore we will now present the OME's expressed in renormalization group coefficients defined in Ref. [3]. The explicit formulas for the OME's can be found in the Appendix and contain terms which are finite in the limit $\varepsilon \rightarrow 0$. Further it is implicitly understood that all quantities in this section, in particular the anomalous dimensions $\gamma_{i j}(i, j$ $=q, g)$, are Mellin transforms even though we suppress the moment index $n$ to simplify the notation.

[Another way to interpret the formulas is that the OME's are given in parton momentum fraction $(z)$ space when the anomalous dimensions are replaced by minus the corresponding Altarelli-Parisi splitting functions and the multiplications are replaced by convolutions.] We have written the OME's in such a way that all renormalization group coefficients appearing in the expressions below are given in the $\overline{\mathrm{MS}}$ scheme.

In the rest of the section the superscript 5 will be suppressed because we will only discuss the spin OME's. Up to order $\alpha_{s}^{2}$ the nonsinglet and the singlet OME's can be decomposed into

$$
\hat{A}_{q q}^{r}=\left[\gamma_{5} \Delta \hat{A}_{q q}^{r, \text { phys }}+\gamma_{5} p \frac{\Delta \cdot p}{p^{2}} \hat{A}_{q q}^{r, \mathrm{EOM}}\right](\Delta \cdot p)^{n-1},
$$

where $A_{q q}^{r, \text { phys }}$ and $A_{q q}^{r \text {,EOM }}$ stand for the physical and unphysical parts, respectively, with $r=\mathrm{NS}, \mathrm{S}$. The presence of the latter is due to the fact that the equations of motion are not satisfied.

The nonsinglet physical OME can now be expressed in renormalization group coefficients as follows: 


$$
\begin{aligned}
& \hat{A}_{q q}^{\mathrm{NS}, \text { phys }}=1+\hat{a}_{s} S_{\varepsilon}\left(\frac{-p^{2}}{\mu^{2}}\right)^{\varepsilon / 2}\left[\frac{1}{\varepsilon} \gamma_{q q}^{\mathrm{NS},(0)}+a_{q q}^{\mathrm{NS},(1)}-z_{q q}^{(1)}\right. \\
& \left.+\varepsilon\left(a_{q q}^{\mathrm{NS}, \varepsilon,(1)}-z_{q q}^{\varepsilon,(1)}\right)\right] \\
& +\hat{a}_{s}^{2} S_{\varepsilon}^{2}\left(\frac{-p^{2}}{\mu^{2}}\right)^{\varepsilon}\left[\frac{1}{\varepsilon^{2}}\left\{\frac{1}{2}\left(\gamma_{q q}^{\mathrm{NS},(0)}\right)^{2}-\beta_{0} \gamma_{q q}^{\mathrm{NS},(0)}\right\}\right. \\
& +\frac{1}{\varepsilon}\left\{\frac{1}{2} \gamma_{q q}^{\mathrm{NS},(1)}-2 \beta_{0} z_{q q}^{(1)}-2 \beta_{0}\left(a_{q q}^{\mathrm{NS},(1)}-z_{q q}^{(1)}\right)\right. \\
& \left.+\gamma_{q q}^{\mathrm{NS},(0)}\left(a_{q q}^{\mathrm{NS},(1)}-z_{q q}^{(1)}\right)-\hat{\xi} \frac{d\left(a_{q q}^{\mathrm{NS},(1)}-z_{q q}^{(1)}\right)}{d \hat{\xi}} z_{\xi}\right\} \\
& +a_{q q}^{\mathrm{NS},(2)}-z_{q q}^{\mathrm{NS},(2)}-z_{q q}^{(1)}\left(a_{q q}^{\mathrm{NS},(1)}-z_{q q}^{(1)}\right) \\
& -2 \beta_{0}\left(a_{q q}^{\mathrm{NS}, \varepsilon,(1)}-z_{q q}^{\varepsilon,(1)}\right)+\gamma_{q q}^{\mathrm{NS},(0)}\left(a_{q q}^{\mathrm{NS}, \varepsilon,(1)}-z_{q q}^{\varepsilon,(1)}\right) \\
& \left.-\hat{\xi} \frac{d\left(a_{q q}^{\mathrm{NS}, \varepsilon,(1)}-z_{q q}^{\varepsilon,(1)}\right)}{d \hat{\xi}} z_{\xi}\right]_{\hat{\xi}=1} .
\end{aligned}
$$

The $\gamma_{i j}^{(k)}$ denote the coefficients of the order $a_{s}^{k+1}$ terms appearing in the series expansions for the anomalous dimensions. Using the same notation we can also express the unphysical part of the nonsinglet OME in Eq. (2.17) in the aforementioned renormalization group coefficients

$$
\begin{aligned}
\hat{A}_{q q}^{\mathrm{NS}, \mathrm{EOM}}= & \hat{a}_{s} S_{\varepsilon}\left(\frac{-p^{2}}{\mu^{2}}\right)^{\varepsilon / 2}\left[b_{q q}^{\mathrm{NS},(1)}+\varepsilon b_{q q}^{\mathrm{NS}, \varepsilon,(1)}\right] \\
& +\hat{a}_{s}^{2} S_{\varepsilon}^{2}\left(\frac{-p^{2}}{\mu^{2}}\right)^{\varepsilon}\left[\frac { 1 } { \varepsilon } \left\{\gamma_{q q}^{\mathrm{NS},(0)} b_{q q}^{\mathrm{NS},(1)}-2 \beta_{0} b_{q q}^{\mathrm{NS},(1)}\right.\right. \\
& \left.-\hat{\xi} \frac{d b_{q q}^{\mathrm{NS},(1)}}{d \hat{\xi}} z_{\xi}\right\}+b_{q q}^{\mathrm{NS},(2)}-2 \beta_{0} b_{q q}^{\mathrm{NS}, \varepsilon,(1)} \\
& +\gamma_{q q}^{\mathrm{NS},(0)} b_{q q}^{\mathrm{NS}, \varepsilon,(1)}-z_{q q}^{(1)} b_{q q}^{\mathrm{NS},(1)} \\
& \left.-\hat{\xi} \frac{d b_{q q}^{\mathrm{NS}, \varepsilon,(1)}}{d \hat{\xi}} z_{\xi}\right]_{\hat{\xi}=1}
\end{aligned}
$$

The singlet OME can be decomposed as follows:

$$
\hat{A}_{q q}^{\mathrm{S}}=\hat{A}_{q q}^{\mathrm{NS}}+\hat{A}_{q q}^{\mathrm{PS}},
$$

where the purely singlet physical part equals

$$
\begin{aligned}
\hat{A}_{q q}^{\mathrm{PS}, \text { phys }}= & \hat{a}_{s}^{2} S_{\varepsilon}^{2}\left(\frac{-p^{2}}{\mu^{2}}\right)^{\varepsilon}\left[\frac{1}{\varepsilon^{2}}\left\{\frac{1}{2} \gamma_{q g}^{(0)} \gamma_{g q}^{(0)}\right\}+\frac{1}{\varepsilon}\left\{\frac{1}{2} \gamma_{q q}^{\mathrm{PS},(1)}\right.\right. \\
& \left.\left.+\gamma_{q g}^{(0)} a_{g q}^{(1)}\right\}+a_{q q}^{\mathrm{PS},(2)}-z_{q q}^{\mathrm{PS},(2)}+\gamma_{q g}^{(0)} a_{g q}^{\varepsilon,(1)}\right]
\end{aligned}
$$

and the purely singlet unphysical part equals

$$
\hat{A}_{q q}^{\mathrm{PS}, \mathrm{EOM}}=\hat{a}_{s}^{2} S_{\varepsilon}^{2}\left(\frac{-p^{2}}{\mu^{2}}\right)^{\varepsilon}\left[\frac{1}{\varepsilon}\left\{\gamma_{q g}^{(0)} b_{g q}^{(1)}\right\}+b_{q q}^{\mathrm{PS},(2)}+\gamma_{q g}^{(0)} b_{g q}^{\varepsilon,(1)}\right] .
$$

The next OME is

$$
\hat{A}_{q g, \mu \nu}=\epsilon_{\mu \nu \alpha \beta} \Delta^{\alpha} p^{\beta} \frac{1}{\Delta \cdot p} \hat{A}_{q g}^{\text {phys }},
$$

with

$$
\begin{aligned}
\hat{A}_{q g}^{\mathrm{phys}}= & \hat{a}_{s} S_{\varepsilon}\left(\frac{-p^{2}}{\mu^{2}}\right)^{\varepsilon / 2}\left[\frac{1}{\varepsilon} \gamma_{q g}^{(0)}+a_{q g}^{(1)}+\varepsilon a_{q g}^{\varepsilon,(1)}\right] \\
& +\hat{a}_{s}^{2} S_{\varepsilon}^{2}\left(\frac{-p^{2}}{\mu^{2}}\right)^{\varepsilon}\left[\frac { 1 } { \varepsilon ^ { 2 } } \left\{\frac{1}{2}\left(\gamma_{q q}^{(0)} \gamma_{q g}^{(0)}+\gamma_{q g}^{(0)} \gamma_{g g}^{(0)}\right)\right.\right. \\
& \left.-\beta_{0} \gamma_{q g}^{(0)}\right\}+\frac{1}{\varepsilon}\left\{\frac{1}{2}\left(\gamma_{q g}^{(1)}-z_{q q}^{(1)} \gamma_{q g}^{(0)}\right)-2 \beta_{0} a_{q g}^{(1)}\right. \\
& \left.+\gamma_{q g}^{(0)} a_{g g}^{(1)}+\gamma_{q q}^{(0)} a_{q g}^{(1)}-\hat{\xi} \frac{\partial a_{q g}^{(1)}}{d \hat{\xi}} z_{\xi}\right\}+a_{q g}^{(2)}-2 \beta_{0} a_{q g}^{\varepsilon,(1)} \\
& +\gamma_{q q}^{(0)} a_{q g}^{\varepsilon,(1)}+\gamma_{q g}^{(0)} a_{g g}^{\varepsilon,(1)}-z_{q q}^{(1)} a_{q g}^{(1)}-z_{q q}^{\varepsilon,(1)} \gamma_{q g}^{(0)} \\
& \left.-\hat{\xi} \frac{d a_{q g}^{\varepsilon,(1)}}{d \hat{\xi}} z_{\xi}\right]_{\hat{\xi}=1} .
\end{aligned}
$$

Next we need

$$
\hat{A}_{g q}=\left[\gamma_{5} \Delta \hat{A}_{g q}^{\mathrm{phys}}+\gamma_{5} p \frac{\Delta \cdot p}{p^{2}} \hat{A}_{g q}^{\mathrm{EOM}}\right](\Delta \cdot p)^{n-1},
$$

where

$$
\begin{aligned}
\hat{A}_{g q}^{\text {phys }}= & \hat{a}_{s} S_{\varepsilon}\left(\frac{-p^{2}}{\mu^{2}}\right)^{\varepsilon / 2}\left[\frac{1}{\varepsilon} \gamma_{g q}^{(0)}+a_{g q}^{(1)}+\varepsilon a_{g q}^{\varepsilon,(1)}\right] \\
& +\hat{a}_{s}^{2} S_{\varepsilon}^{2}\left(\frac{-p^{2}}{\mu^{2}}\right)^{\varepsilon}\left[\frac { 1 } { \varepsilon ^ { 2 } } \left\{\frac{1}{2}\left(\gamma_{g g}^{(0)} \gamma_{g q}^{(0)}+\gamma_{g q}^{(0)} \gamma_{q q}^{(0)}\right)\right.\right. \\
& \left.-\beta_{0} \gamma_{g q}^{(0)}\right\}+\frac{1}{\varepsilon}\left\{\frac{1}{2}\left(\gamma_{g q}^{(1)}+\gamma_{g q}^{(0)} z_{q q}^{(1)}\right)-2 \beta_{0} a_{g q}^{(1)}+\gamma_{g q}^{(0)}\right. \\
& \left.\times\left(a_{q q}^{(1)}-z_{q q}^{(1)}\right)+\gamma_{g g}^{(0)} a_{g q}^{(1)}-\hat{\xi} \frac{d a_{g q}^{(1)}}{d \hat{\xi}} z_{\xi}\right\}+a_{g q}^{(2)} \\
& -2 \beta_{0} a_{g q}^{\varepsilon,(1)}+\gamma_{g q}^{(0)} z_{q q}^{\varepsilon,(1)}+\gamma_{g q}^{(0)}\left(a_{q q}^{\varepsilon,(1)}-z_{q q}^{\varepsilon,(1)}\right) \\
& \left.+\gamma_{g g}^{(0)} a_{g q}^{\varepsilon,(1)}-\hat{\xi} \frac{d a_{g q}^{\varepsilon,(1)}}{d \hat{\xi}} z_{\xi}\right]_{\hat{\xi}=1}
\end{aligned}
$$

and 


$$
\begin{aligned}
\hat{A}_{g q}^{\mathrm{EOM}}= & \hat{a}_{s} S_{\varepsilon}\left(\frac{-p^{2}}{\mu^{2}}\right)^{\varepsilon / 2}\left[b_{g q}^{(1)}+\varepsilon b_{g q}^{\varepsilon,(1)}\right] \\
& +\hat{a}_{s}^{2} S_{\varepsilon}^{2}\left(\frac{-p^{2}}{\mu^{2}}\right)^{\varepsilon}\left[\frac { 1 } { \varepsilon } \left\{\gamma_{g q}^{(0)} b_{q q}^{(1)}+\gamma_{g g}^{(0)} b_{g q}^{(1)}-2 \beta_{0} b_{g q}^{(1)}\right.\right. \\
& \left.-\hat{\xi} \frac{d b_{g q}^{(1)}}{d \hat{\xi}} z_{\xi}\right\}+b_{g q}^{(2)}-2 \beta_{0} b_{g q}^{\varepsilon,(1)}+\gamma_{g q}^{(0)} b_{q q}^{\mathrm{NS}, \varepsilon,(1)} \\
& \left.+\gamma_{g g}^{(0)} b_{g q}^{\varepsilon,(1)}-\hat{\xi} \frac{d b_{g q}^{\mathrm{NS}, \varepsilon,(1)}}{d \hat{\xi}} z_{\xi}\right]_{\hat{\xi}=1} .
\end{aligned}
$$

Finally we need

$$
\hat{A}_{g g, \mu \nu}=\epsilon_{\mu \nu \alpha \beta} \Delta^{\alpha} p^{\beta} \frac{1}{\Delta \cdot p} \hat{A}_{g g}^{\text {phys }},
$$

with

$$
\begin{aligned}
\hat{A}_{g g}^{\text {phys }}= & 1+\hat{a}_{s} S_{\varepsilon}\left(\frac{-p^{2}}{\mu^{2}}\right)^{\varepsilon / 2}\left[\frac{1}{\varepsilon} \gamma_{g g}^{(0)}+a_{g g}^{(1)}+\varepsilon a_{g g}^{\varepsilon,(1)}\right] \\
& +\hat{a}_{s}^{2} S_{\varepsilon}^{2}\left(\frac{-p^{2}}{\mu^{2}}\right)^{\varepsilon}\left[\frac{1}{\varepsilon^{2}}\left\{\frac{1}{2}\left(\left(\gamma_{g g}^{(0)}\right)^{2}+\gamma_{g q}^{(0)} \gamma_{q g}^{(0)}\right)-\beta_{0} \gamma_{g g}^{(0)}\right\}\right. \\
& +\frac{1}{\varepsilon}\left\{\frac{1}{2} \gamma_{g g}^{(1)}-2 \beta_{0} a_{g g}^{(1)}+\gamma_{g g}^{(0)} a_{g g}^{(1)}+\gamma_{g q}^{(0)} a_{q g}^{(1)}\right. \\
& \left.-\hat{\xi} \frac{d a_{g g}^{(1)}}{d \hat{\xi}} z_{\xi}\right\}+a_{g g}^{(2)}-2 \beta_{0} a_{g g}^{\varepsilon,(1)}+\gamma_{g g}^{(0)} a_{g g}^{\varepsilon,(1)} \\
& \left.+\gamma_{g q}^{(0)} a_{q g}^{\varepsilon,(1)}-\hat{\xi} \frac{d a_{g g}^{\varepsilon,(1)}}{d \hat{\xi}} z_{\xi}\right]_{\hat{\xi}=1} .
\end{aligned}
$$

Explicit formulas for the above unrenormalized OME's corrected up to second order, which contain all the finite terms independent of $\varepsilon$, are given in Appendix A. The pole terms agree with the results in Eqs. (2.18)-(2.29). Since the external quark and gluon legs are off-shell one can directly insert the formulas in Appendix A into Feynman integrals for one-loop graphs.

In this way one gets results for subsets of the three-loop graphs. It is, however, clear that the most difficult Feynman integrals containing nonplanar diagrams, where all quark or gluon lines cross, remain to be done. The renormalized OME's are given by

$$
\begin{aligned}
& A_{q q}^{\mathrm{NS}}=Z_{q q}^{5, \mathrm{NS}}\left(Z^{-1}\right)_{q q}^{\mathrm{NS}} \hat{A}_{q q}^{\mathrm{NS}}, \\
& A_{q q}=Z_{q q}^{5, \mathrm{~S}}\left(Z^{-1}\right)_{q q}^{\mathrm{S}} \hat{A}_{q q}+\left(Z^{-1}\right)_{q g} \hat{A}_{g q}, \\
& A_{q g}=Z_{q q}^{5, \mathrm{~S}}\left(Z^{-1}\right)_{q q}^{\mathrm{S}} \hat{A}_{q g}+\left(Z^{-1}\right)_{q g} \hat{A}_{g g}, \\
& A_{g q}=Z_{q q}^{5, \mathrm{~S}}\left(Z^{-1}\right)_{g q} \hat{A}_{q q}+\left(Z^{-1}\right)_{g g} \hat{A}_{g q},
\end{aligned}
$$

and

$$
A_{g g}=Z_{q q}^{5, \mathrm{~S}}\left(Z^{-1}\right)_{g q} \hat{A}_{q g}+\left(Z^{-1}\right)_{g g} \hat{A}_{g g} .
$$

The operator renormalization constants are given by

$$
\left(Z^{-1}\right)_{q q}^{\mathrm{S}}=\left(Z^{-1}\right)_{q q}^{\mathrm{NS}}+\left(Z^{-1}\right)_{q q}^{\mathrm{PS}},
$$

where

$$
\begin{aligned}
\left(Z^{-1}\right)_{q q}^{\mathrm{NS}}= & 1+a_{s} S_{\varepsilon}\left[-\frac{1}{\varepsilon} \gamma_{q q}^{(0)}\right]+a_{s}^{2} S_{\varepsilon}^{2}\left[\frac { 1 } { \varepsilon ^ { 2 } } \left\{\frac{1}{2}\left(\gamma_{q q}^{(0)}\right)^{2}\right.\right. \\
& \left.\left.-\beta_{0} \gamma_{q q}^{(0)}\right\}-\frac{1}{2 \varepsilon} \gamma_{q q}^{\mathrm{NS},(1)}\right]
\end{aligned}
$$

and

$$
\left(Z^{-1}\right)_{q q}^{\mathrm{PS}}=a_{s}^{2} S_{\varepsilon}^{2}\left[\frac{1}{\varepsilon^{2}}\left\{\frac{1}{2} \gamma_{q g}^{(0)} \gamma_{g q}^{(0)}\right\}-\frac{1}{2 \varepsilon} \gamma_{q q}^{\mathrm{PS},(1)}\right] .
$$

The other singlet operator renormalization constants are given by

$$
\begin{aligned}
\left(Z^{-1}\right)_{q g}= & a_{s} S_{\varepsilon}\left[-\frac{1}{\varepsilon} \gamma_{q g}^{(0)}\right]+a_{s}^{2} S_{\varepsilon}^{2}\left[\frac { 1 } { \varepsilon ^ { 2 } } \left\{\frac{1}{2}\left(\gamma_{q q}^{(0)} \gamma_{q g}^{(0)}+\gamma_{q g}^{(0)} \gamma_{g g}^{(0)}\right)\right.\right. \\
& \left.\left.-\beta_{0} \gamma_{q g}^{(0)}\right\}-\frac{1}{2 \varepsilon}\left\{\gamma_{q g}^{(1)}+z_{q q}^{(1)} \gamma_{q g}^{(0)}\right\}\right], \\
\left(Z^{-1}\right)_{g q}= & a_{S} S_{\varepsilon}\left[-\frac{1}{\varepsilon} \gamma_{g q}^{(0)}\right]+a_{s}^{2} S_{\varepsilon}^{2}\left[\frac { 1 } { \varepsilon ^ { 2 } } \left\{\frac{1}{2}\left(\gamma_{g q}^{(0)} \gamma_{q q}^{(0)}+\gamma_{g g}^{(0)} \gamma_{g q}^{(0)}\right)\right.\right. \\
& \left.\left.-\beta_{0} \gamma_{g q}^{(0)}\right\}-\frac{1}{2 \varepsilon}\left\{\gamma_{g q}^{(1)}-z_{q q}^{(1)} \gamma_{g q}^{(0)}\right\}\right],
\end{aligned}
$$

and

$$
\begin{aligned}
\left(Z^{-1}\right)_{g g}= & 1+a_{s} S_{\varepsilon}\left[-\frac{1}{\varepsilon} \gamma_{g g}^{(0)}\right] \\
& +a_{s}^{2} S_{\varepsilon}^{2}\left[\frac{1}{\varepsilon^{2}}\left\{\frac{1}{2}\left(\left(\gamma_{g g}^{(0)}\right)^{2}+\gamma_{g q}^{(0)} \gamma_{q g}^{(0)}\right)-\beta_{0} \gamma_{g g}^{(0)}\right\}\right. \\
& \left.-\frac{1}{2 \varepsilon} \gamma_{g g}^{(1)}\right]
\end{aligned}
$$

The above renormalization constants are chosen in such a way that the anomalous dimensions $\gamma_{i j}^{(1)}$ are presented in the MS scheme. Naively one would expect that the single pole terms in Eqs. (2.38) and (2.39) are then given by $-\gamma_{q g}^{(1)} / 2$ and $-\gamma_{g q}^{(1)} / 2$, respectively. However, due to the HVBM prescription the renormalization constants $Z_{i j}$ have to be modified in order to bring the renormalized OME's in the stan- 
dard form. We have checked that the latter satisfy the renormalization group equations with the anomalous dimensions $\gamma_{i j}^{(k)}$.

Notice that only the renormalized OME's are independent of the prescription for the $\gamma_{5}$ matrix and the Levi-Civita tensor.

To conclude we discuss properties of the renormalization factors $Z_{q q}^{5, r}, r=\mathrm{NS}, \mathrm{S}$ in Eq. (2.8) computed in this paper and compare them with other results in the literature. Starting with the nonsinglet term, which is computed via Eq. (2.15), the unrenormalized expression can be found in Eq. (A11). Due to the factor $(-1)^{n}$ [for its origin see below Eq. (A3)] it can be split into two parts as follows

$$
Z_{q q}^{5, \mathrm{NS}}=\int_{0}^{1} d z z^{n-1}\left[Z_{q q}^{5, \mathrm{NS},(+)}(z)+(-1)^{n} Z_{q q}^{5, \mathrm{NS},(-)}(z)\right] .
$$

After coupling constant and gauge constant renormalization [see Eqs. (2.11), (2.12)] the two parts become

$$
\begin{aligned}
Z_{q q}^{5, \mathrm{NS},(+)}(z)= & \delta(1-z)+a_{s} S_{\varepsilon} C_{F}[-8(1-z) \\
& +\varepsilon\{-4(1-z) \ln (1-z)-4(1-z) \ln z \\
& +2 z-(1-\xi)\}]+a_{s}^{2} S_{\varepsilon}^{2}\left[C_{F}^{2}\{-16(1-z)\right. \\
& -(16+8 z) \ln z+16(1-z) \ln z \ln (1-z)\} \\
& +C_{A} C_{F}\left\{-\frac{1}{\varepsilon}\left(\frac{88}{3}(1-z)\right)-\frac{592}{9}(1-z)\right. \\
& +8(1-z) \zeta(2)+\left(-\frac{80}{3}+\frac{8}{3} z\right) \ln z \\
& \left.-4(1-z) \ln ^{2} z\right\}+n_{f} C_{F} T_{f}\left\{\frac{1}{\varepsilon}\left(\frac{32}{3}(1-z)\right)\right. \\
& \left.\left.+\frac{80}{9}(1-z)+\frac{16}{3}(1-z) \ln z\right\}\right)
\end{aligned}
$$

and

$$
\begin{aligned}
Z_{q q}^{5, \mathrm{NS},(-)}(z)= & a_{s}^{2} S_{\varepsilon}^{2}\left[( C _ { F } ^ { 2 } - \frac { 1 } { 2 } C _ { A } C _ { F } ) \left\{8 ( 1 + z ) \left[4 \mathrm{Li}_{2}(-z)\right.\right.\right. \\
& \left.+4 \ln z \ln (1+z)+2 \zeta(2)-\ln ^{2} z-3 \ln z\right] \\
& -56(1-z)\}]
\end{aligned}
$$

respectively. Choosing $n=1$ in Eq. (2.41) we obtain

$$
\begin{aligned}
Z_{q q}^{5, \mathrm{NS}}= & 1+a_{s} S_{\varepsilon} C_{F}\{-4+\varepsilon[5-(1-\xi)]\} \\
& +a_{s}^{2} S_{\varepsilon}^{2}\left[C_{F}^{2}\{22\}+C_{A} C_{F}\left\{-\frac{44}{3} \frac{1}{\varepsilon}-\frac{107}{9}\right\}\right. \\
& \left.+n_{f} C_{F} T_{f}\left\{\frac{16}{3} \frac{1}{\varepsilon}+\frac{4}{9}\right\}\right] .
\end{aligned}
$$

This is the renormalization constant which has to be multiplied with the nonsinglet axial-vector current $O_{q, k}^{5, \mu}$ in Eq.
(2.1) so that the latter will not be renormalized (i.e., remain a conserved current) if one employs the HVBM prescription.

This renormalization constant has been calculated up to order $\alpha_{s}^{3}$ in Refs. [22,23]. Except for the term proportional to $\varepsilon$, which was not presented previously, our result in Eq. (2.44) agrees with that in the aforementioned literature.

Notice that there is a difference in notation between the renormalization constants defined in our paper and those in Refs. [22,23]. First the $\varepsilon$ in the previous papers is equal to $-\varepsilon / 2$ in our work.

Second a comparison between Eqs. (2.30), (2.31) and Eqs. (5), (11) in Ref. [22] reveals the relation $Z_{q q}^{5, r}\left(Z^{-1}\right)_{q q}^{r}$ $=Z_{5}^{r} Z_{\mathrm{MS}}^{r}(r=\mathrm{NS}, \mathrm{S})$. For the nonsinglet axial vector current $(r=\mathrm{NS})$, which has the property $\left(Z^{-1}\right)_{q q}^{\mathrm{NS}}=1, Z_{\mathrm{MS}}^{\mathrm{NS}}$ and $Z_{5}^{\mathrm{NS}}$ are given in Eqs. (8) and (11) of Ref. [23], respectively. For $Z_{5}^{\mathrm{NS}}$ see also Eq. (9) in Ref. [22]. Notice that in Ref. [23] $Z_{\mathrm{MS}}^{\mathrm{NS}}$ and $Z_{5}^{\mathrm{NS}}$ are called $Z_{A}$ and $Z_{5}$, respectively.

Recently the factor $Z_{q q}^{5 \text {,NS }}$ has been also computed in Ref. [25] for the coefficient functions corresponding to the longitudinal spin fragmentation function in $e^{+} e^{-}$scattering. If we indicate the latter factor by the superscript $\mathrm{T}$ (timelike) and the one in Eq. (2.41) by S (spacelike) we have the following relation

$$
Z_{q q}^{5, \mathrm{NS},(+), \mathrm{T}}(z)=-z Z_{q q}^{5, \mathrm{NS},(+), \mathrm{S}}\left(\frac{1}{z}\right)+a_{s}^{2}\left[\beta_{0} z_{q q}^{\mathrm{NS},(1)}(z) \ln z\right]
$$

This result demonstrates the breakdown of the GribovLipatov relation [26] which is therefore only valid up to order $\alpha_{s}$.

However for the minus component

$$
Z_{q q}^{5, \mathrm{NS},(-), \mathrm{T}}(z)=-z Z_{q q}^{5, \mathrm{NS},(-), \mathrm{S}}\left(\frac{1}{z}\right)
$$

so the Gribov-Lipatov relation still holds at least up to second order. The above relations have also been reported for the spin averaged nonsinglet spacelike $(\mathrm{S})$ and timelike $(\mathrm{T})$ splitting functions $P_{q q}^{\mathrm{NS}}$ in Eqs. (6.37) and (6.38) of Ref. [27] where $z_{q q}^{(1)}$ is replaced by $P_{q q}^{(0)}$. Notice that there is no difference between spin and spin averaged splitting functions as far as the nonsinglet part is concerned (see Ref. [28]). However, there is a difference between $Z_{q q}^{5, \mathrm{NS}}$ and $P_{q q}^{\mathrm{NS}}$ if one looks at relation (6.46) in Ref. [27]. In our case it reads

$$
\begin{aligned}
Z_{q q}^{5, \mathrm{NS},(+), \mathrm{T}}(z)= & Z_{q q}^{5, \mathrm{NS},(+), \mathrm{S}}(z) \\
& +a_{s}^{2} \frac{1}{2} \ln z \int_{z}^{1} d y\left[z_{q q}^{\mathrm{NS},(1)}\left(\frac{z}{y}\right) P_{q q}^{(0)}(y)\right],
\end{aligned}
$$


whereas in Eq. (6.46) of Ref. [27] the $\ln z$ is shifted to the integrand where it becomes $\ln y$. Hence the first moments of $Z_{q q}^{5, \mathrm{NS}, \mathrm{T}}$ and $Z_{q q}^{5, \mathrm{NS}, \mathrm{S}}$ are not equal anymore. It implies that the coefficient 22 of the $C_{F}^{2}$ part of $Z_{q q}^{5, \mathrm{NS}, \mathrm{S}}$ in Eq. (2.44) is changed into $12+16 \zeta(2)$ for $Z_{q q}^{5, \mathrm{NS}, \mathrm{T}}$ (see Eq. (3.46) in Ref. [25]). Note that the Gribov-Lipatov relation originated from a study of conformal invariant field theories. However, conformal invariance is broken in renormalized perturbation theories such as QCD due to the appearance of the renormalization scale which enters in the renormalized coupling constant $a_{s}(\mu)$.

Therefore there is no reason to expect that the GribovLipatov relation holds in higher order but all the breaking terms should be proportional to the $\beta$ function whose lowest order coefficient in the perturbation series is given in Eq. (2.45).

In some supersymmetric theories where the coupling constant is not renormalized the beta-function equals zero so that the conformal invariance is preserved in all orders of perturbation theory.

The calculation of $Z_{q q}^{\mathrm{PS},(2)}$, which can only come from $\hat{A}_{q q}^{\mathrm{PS} \text { phys }}$ in Eq. (2.21), proceeds as follows. Up to second order only one diagram contributes to this OME, which can be found in Fig. 3.1 of [3]. Following the same arguments as given below Eq. (2.15) one has to anticommute the $\gamma_{5}$ matrix to another vertex in the diagram so that $Z_{q q}^{5, \mathrm{PS}}=1$. However, in this procedure the Adler-Bell-Jackiw (ABJ) anomaly, characteristic of this graph, will also then be removed. In this case the expression for Eq. (2.21) becomes

$$
\begin{aligned}
\hat{A}_{q q}^{\mathrm{PS}, \text { phys, } \mathrm{ABJ}}= & \hat{a}_{s}^{2} S_{\varepsilon}^{2}\left(\frac{-p^{2}}{\mu^{2}}\right)^{\varepsilon}\left[\frac{1}{\varepsilon^{2}}\left\{\frac{1}{2} \gamma_{q g}^{(0)} \gamma_{g q}^{(0)}\right\}\right. \\
& +\frac{1}{\varepsilon}\left\{\frac{1}{2}\left(\gamma_{q q}^{\mathrm{PS},(1)}+v_{q g}^{(1)} \gamma_{g q}^{(0)}\right)+\gamma_{q g}^{(0)} a_{g q}^{(1)}\right\} \\
& \left.+a_{q q}^{\mathrm{PS},(2)}+v_{q g}^{(1)} a_{g q}^{(1)}+\gamma_{q g}^{(0)} a_{g q}^{\varepsilon,(1)}\right] .
\end{aligned}
$$

Comparing the above expression with the one in Eq. (2.21) we observe that in the former the effective anomalous dimension has become $\gamma_{q q}^{\mathrm{PS},(1)}+v_{q g}^{(1)} \gamma_{g q}^{(0)}$ with $v_{q g}^{(1)}=8 T_{f}(1$ $-z$ ). If we take the first moment of this expression this effective anomalous dimension becomes zero meaning that the $\mathrm{ABJ}$ anomaly vanishes.

Therefore there is a striking analogy between $v_{q g}^{(1)}$ above and $z_{q q}^{(1)}$ in Eq. (2.8). Both constants remove anomalies i.e. the former in the case of the singlet current $O_{q}^{5, \mu}$ and the latter in the case of the nonsinglet current $O_{q, k}^{5, \mu}$. However, the ABJ anomaly has to be restored. Hence from Eqs. (2.21), (2.26), and (2.48) we get

$$
\begin{aligned}
\hat{A}_{q q}^{\mathrm{PS}, \text { phys, } \mathrm{ABJ}}-\hat{A}_{q q}^{\mathrm{PS}, \text { phys }}-\hat{a}_{s} S_{\varepsilon}\left[v_{q g}^{(1)}\right] A_{g q}^{\mathrm{phys}} \\
+\hat{a}_{s}^{2} S_{\varepsilon}^{2}\left[\frac{1}{2 \varepsilon}\left(v_{q g}^{(1)} \gamma_{g q}^{(0)}\right)\right]=\hat{a}_{s}^{2} S_{\varepsilon}^{2}\left[z_{q q}^{\mathrm{PS},(2)}\right],
\end{aligned}
$$

where the last term before the equal sign restores the $\mathrm{ABJ}$ anomaly in $\hat{A}_{q q}^{\mathrm{PS} \text {, phys, } \mathrm{ABJ}}$. Finally we obtain

$$
z_{q q}^{\mathrm{PS},(2)}=n_{f} C_{F} T_{f}\left[16(1-z)+8(3-z) \ln z+4(2+z) \ln ^{2} z\right] .
$$

If one takes the first moment of this expression the result becomes $3 n_{f} C_{F} T_{f}$. This number has to be added to the nonsinglet expression in Eq. (2.44) to obtain the singlet renormalization constant $Z_{q q}^{5, \mathrm{~S}}$ in Eq. (2.13). The latter can be compared with $Z_{\mathrm{MS}}^{\mathrm{S}}$ and $Z_{5}^{\mathrm{S}}$ given in Eqs. (12) and (23), respectively, of Ref. [22]. Using the relations between our notations for the renormalization constants and the ones in Ref. [22], which are given below Eq. (2.44), we find complete agreement with Ref. [22]. Notice that in the singlet case the axial vector current is not conserved anymore because of the ABJ anomaly. This is revealed by the anomalous dimension $\gamma_{q q}^{\mathrm{PS},(1)}=24 n_{f} C_{F} T_{f}$ in Eq. (2.37) so that we get $\left(Z^{-1}\right)_{q q}^{\mathrm{S}}=1+a_{s}^{2} S_{\varepsilon}^{2}\left[-12 n_{f} C_{F} T_{f} / \varepsilon\right]$.

Finally one could ask why it is preferable to choose the HVBM instead of the naive $\gamma_{5}$ prescription since in the latter case $Z_{q q}^{5, r}=1$. The first reason is that HVBM was originally proposed to obtain the $\mathrm{ABJ}$ anomaly in the fermion triangle graph, which is not always correctly reproduced using the naive prescription [see Eq. (2.48)]. The second reason is that the Levi-Civita tensor appearing in the OME $A_{g q}$ induces the HVBM prescription in the subgraphs containing quark loops. Therefore the naive $\gamma_{5}$ prescription does not prevent an additional renormalization so it is inconsistent. It is better to use a consistent procedure like the HVBM where all constants are fixed once and for all.

\section{ACKNOWLEDGMENTS}

This research was supported in part by the National Science Foundation Grant No. PHY-9722101.

\section{APPENDIX}

In this appendix we present complete expressions for the two-loop corrected OME's computed from the Feynman diagrams depicted in Ref. [3]. The second order contributions are calculated up to finite terms which survive in the limit $\varepsilon \rightarrow 0$. The OME's presented here are unrenormalized and external self-energy corrections are included. In these expressions definitions of the Riemann zeta-functions $\zeta(n)$ and the polylogarithms $\operatorname{Li}_{n}(z), \mathrm{S}_{n, m}(z)$ can be found in Ref. [29]. Also the distributions $[1 /(1-z)]_{+}$and $[\ln (1-z) /(1-z)]_{+}$ and written as $1 /(1-z)$ and $\ln (1-z) /(1-z)$, respectively, to shorten the formulas. Note that the OME's given in the text are the moments of the functions listed here so

$$
A_{i j}^{n}=\int_{0}^{1} d z z^{n-1} A_{i j}\left(z, \frac{-p^{2}}{\mu^{2}}, \frac{1}{\varepsilon}\right),
$$

where for simplicity we have not written the moment index $n$ on the functions. Also to simplify the expressions we define the phase-space factor 


$$
F=\frac{\hat{\alpha}_{s}}{4 \pi} S_{\varepsilon}\left(\frac{-p^{2}}{\mu^{2}}\right)^{\varepsilon / 2} .
$$

We first split $\hat{A}_{q q}^{\mathrm{NS}}$ into physical and unphysical parts following the notation in Eq. (2.17). The physical part is [see also Eq. (2.18)]

$$
\begin{aligned}
& \hat{A}_{q q}^{\mathrm{NS}, \text { phys }}\left(z, \frac{-p^{2}}{\mu^{2}}, \frac{1}{\varepsilon}\right)=\delta(1-z)+F C_{F}\left[\frac{1}{\varepsilon}\left\{-4-4 z+\frac{8}{1-z}+6 \delta(1-z)\right\}-4+2 z+2\left(\frac{2}{1-z}-1-z\right)\right. \\
& \times[\ln z+\ln (1-z)]-\delta(1-z)[7-4 \zeta(2)]+\frac{1-\hat{\xi}}{1-z}+\varepsilon\{1-(2-z)[\ln z+\ln (1-z)] \\
& +\frac{1}{2}\left(\frac{2}{1-z}-1-z\right)\left[\zeta(2)+\{\ln z+\ln (1-z)\}^{2}\right]+\delta(1-z)\left[7-\frac{3}{4} \zeta(2)-4 \zeta(3)\right] \\
& \left.\left.+\frac{1}{2}(1-\hat{\xi})\left(-\frac{1}{1-z}+\frac{\ln (1-z)}{1-z}+\frac{\ln z}{1-z}+\delta(1-z)\{-2+\zeta(2)\}\right)\right\}\right] \\
& +F^{2}\left(\frac { 1 } { \varepsilon ^ { 2 } } \left\{C _ { F } ^ { 2 } \left[40+8 z-\frac{48}{1-z}-2 \delta(1-z)[9-16 \zeta(2)]+32 \ln (1-z)\left(1+z-\frac{2}{1-z}\right)\right.\right.\right. \\
& \left.-8 \ln z\left(3+3 z-\frac{4}{1-z}\right)\right]+C_{A} C_{F}\left[\frac{44}{3}+\frac{44}{3} z-\frac{88}{3} \frac{1}{1-z}-22 \delta(1-z)\right] \\
& \left.+n_{f} C_{F} T_{f}\left[-\frac{16}{3}\left(1+z-\frac{2}{1-z}\right)+8 \delta(1-z)\right]\right\}+\frac{1}{\varepsilon}\left\{C _ { F } ^ { 2 } \left[-56+12 z+\frac{56}{1-z}+\delta(1-z)\right.\right. \\
& \times\left(\frac{87}{2}-36 \zeta(2)-8 \zeta(3)\right)-4 \ln (1-z)\left(-15+5 z+\frac{6}{1-z}\right)+24 \ln ^{2}(1-z)\left(1+z-\frac{2}{1-z}\right) \\
& \left.+4 \ln z\left(1+3 z-\frac{9}{1-z}\right)-16 \ln z \ln (1-z) \frac{1}{1-z}-2 \ln ^{2} z\left(7+7 z-\frac{8}{1-z}\right)-8 \operatorname{Li}_{2}(1-z)(1+z)\right] \\
& +C_{A} C_{F}\left[\frac{106}{9}-\frac{242}{9} z+\frac{238}{9} \frac{1}{1-z}+\delta(1-z)\left(\frac{325}{6}-\frac{44}{3} \zeta(2)-12 \zeta(3)\right)\right. \\
& \left.+4\left(1+z-\frac{2}{1-z}\right)\left(\zeta(2)+\frac{11}{3} \ln (1-z)-\frac{1}{2} \ln ^{2} z\right)+\ln z\left(\frac{34}{3}+\frac{34}{3} z-\frac{44}{3} \frac{1}{1-z}\right)\right]+n_{f} C_{F} T_{f} \\
& \left.\times\left[-\frac{8}{9}+\frac{40}{9} z-\frac{56}{9} \frac{1}{1-z}+\delta(1-z)\left(-\frac{58}{3}+\frac{16}{3} \zeta(2)\right)-\frac{8}{3}\{2 \ln (1-z)+\ln z\}\left(1+z-\frac{2}{1-z}\right)\right]\right\} \\
& +C_{F}^{2}\left[\frac{188}{3}-\frac{32}{3} z-\frac{56}{1-z}+\zeta(2)\left(20-4 z+\frac{32}{3} z^{2}-\frac{4}{1-z}\right)+24 \zeta(3)\left(1-z+2 z^{2}-\frac{1}{1-z}\right)\right. \\
& +\delta(1-z)\left(-\frac{541}{8}+\frac{97}{2} \zeta(2)+54 \zeta(3)-\frac{74}{5} \zeta(2)^{2}\right)+\ln (1-z)\left(-78+62 z+\frac{28}{1-z}\right) \\
& +8 \ln (1-z) \zeta(2)\left(1+z-2 z^{2}-\frac{1}{1-z}\right)-\ln ^{2}(1-z)\left(-35+17 z+\frac{6}{1-z}\right) \\
& +\frac{28}{3} \ln ^{3}(1-z)\left(1+z-\frac{2}{1-z}\right)-4 \ln (1-z) \operatorname{Li}_{2}(1-z)\left(3-z+4 z^{2}\right)-\ln z\left(\frac{44}{3}+\frac{92}{3} z-\frac{44}{1-z}\right) \\
& +\ln z \zeta(2)\left(2-14 z+16 z^{2}\right)+2 \ln z \ln (1-z)\left(5+9 z-\frac{10}{1-z}\right)+2 \ln z \ln ^{2}(1-z)\left(3+3 z-\frac{10}{1-z}\right)
\end{aligned}
$$




$$
\begin{aligned}
& -\ln ^{2} z\left(5+7 z+\frac{16}{3} z^{2}-\frac{15}{1-z}\right)-\ln ^{2} z \ln (1-z)\left(6-2 z+8 z^{2}+\frac{4}{1-z}\right)-\ln ^{3} z\left(5+5 z-\frac{16}{3} \frac{1}{1-z}\right) \\
& -8 \ln z \operatorname{Li}_{2}(1-z)\left(1-3 z+4 z^{2}+\frac{2}{1-z}\right)-4 \operatorname{Li}_{2}(1-z)\left(6-12 z+\frac{1}{1-z}\right) \\
& +4 \operatorname{Li}_{3}(1-z)\left(5-7 z+12 z^{2}\right)+\frac{8}{3}\left(9+\frac{1}{z}+12 z+4 z^{2}\right)\left[\ln z \ln (1+z)+\operatorname{Li}_{2}(-z)\right] \\
& \left.-16\left[\ln z \mathrm{Li}_{2}(-z)-2 \mathrm{Li}_{3}(-z)\right]\left(1-\frac{1}{1-z}\right)-8 \mathrm{~S}_{12}(1-z)\left(1-7 z+6 z^{2}+\frac{4}{1-z}\right)\right] \\
& +C_{A} C_{F}\left[-\frac{1}{27}\left(745-800 z+\frac{670}{1-z}\right)-\frac{1}{3} \zeta(2)\left(-23+7 z+\frac{28}{1-z}+16 z^{2}\right)\right. \\
& -2 \zeta(3)\left(10+4 z+12 z^{2}-\frac{17}{1-z}\right)+\delta(1-z)\left(-\frac{7081}{72}+\frac{301}{18} \zeta(2)+28 \zeta(3)+\frac{49}{5} \zeta(2)^{2}\right) \\
& +\frac{2}{9} \ln (1-z)\left(71-148 z+\frac{119}{1-z}\right)+2 \ln (1-z) \zeta(2)\left(1+3 z+4 z^{2}-\frac{5}{1-z}\right)+\left\{\frac{22}{3} \ln ^{2}(1-z)-\ln ^{3} z\right\} \\
& \times\left(1+z-\frac{2}{1-z}\right)-2 \ln z \zeta(2)\left(3+z+4 z^{2}-\frac{5}{1-z}\right)+\frac{1}{6} \ln ^{2} z\left(23+71 z+16 z^{2}-\frac{22}{1-z}\right) \\
& +\frac{1}{9} \ln z\left(38-64 z+\frac{101}{1-z}\right)+\frac{2}{3} \ln z \ln (1-z)\left(14+5 z-\frac{19}{1-z}\right)+4 \ln z \operatorname{Li}_{2}(1-z)\left(2+4 z^{2}-\frac{3}{1-z}\right) \\
& -4 \mathrm{Li}_{2}(1-z)\left(3 z-\frac{1}{1-z}\right)-4\left(3+\frac{1}{3 z}+4 z+\frac{4}{3} z^{2}\right)\left[\ln z \ln (1+z)+\operatorname{Li}_{2}(-z)\right] \\
& +\left(1-z+4 z^{2}-\frac{1}{1-z}\right)\left[\ln ^{2} z \ln (1-z)+2 \ln (1-z) \operatorname{Li}_{2}(1-z)-6 \operatorname{Li}_{3}(1-z)\right] \\
& \left.+8\left[\ln z \operatorname{Li}_{2}(-z)-2 \operatorname{Li}_{3}(-z)\right]\left(1-\frac{1}{1-z}\right)+S_{12}(1-z)\left(14-6 z+24 z^{2}-\frac{14}{1-z}\right)\right] \\
& +n_{f} C_{F} T_{f}\left[\frac{4}{27}\left(20-19 z+\frac{32}{1-z}\right)+\delta(1-z)\left(\frac{569}{18}-\frac{46}{9} \zeta(2)-8 \zeta(3)\right)\right. \\
& +\frac{4}{9}\{2 \ln (1-z)+\ln z\}\left(-1+5 z-\frac{7}{1-z}\right) \\
& \left.-\frac{2}{3}\left[2 \zeta(2)+\{2 \ln (1-z)+\ln z\}^{2}\right]\left(1+z-\frac{2}{1-z}\right)\right]-2(-1)^{n}\left(C_{F}^{2}-\frac{1}{2} C_{A} C_{F}\right) \\
& \times\left(\frac{1}{\varepsilon}\left\{-8+8 z-4 \ln z(1+z)+2\left(-1+z+\frac{2}{1+z}\right)\left[2 \zeta(2)+4 \ln z \ln (1+z)-\ln ^{2} z+4 \operatorname{Li}_{2}(-z)\right]\right\}\right. \\
& +\frac{43}{3}(1-z)-8 \ln (1-z)(1-z)-2 \zeta(2)\left(1+7 z-\frac{8}{3} z^{2}\right)+8\left(z+\frac{1}{1+z}\right) \ln (1+z) \\
& \times\left[\zeta(2)+\ln z \ln (1+z)+2 \mathrm{Li}_{2}(-z)\right]-4(1+z)\left[\ln z \ln (1-z)+\mathrm{Li}_{2}(1-z)+\mathrm{Li}_{3}(-z)\right] \\
& -\frac{1}{3} \ln z(1-11 z)-\ln ^{2} z\left(2-4 z+\frac{8}{3} z^{2}\right)-2 \ln ^{2} z \ln (1+z)\left(3-z-\frac{4}{1+z}\right)-2 \zeta(3)\left(3+z-\frac{2}{1+z}\right) \\
& -\frac{4}{3}\left(12-\frac{1}{z}+9 z-4 z^{2}\right)\left[\ln z \ln (1+z)+\operatorname{Li}_{2}(-z)\right]+8 \mathrm{~S}_{12}(-z)\left(-1+3 z+\frac{4}{1+z}\right) \\
& +4\left(1-z-\frac{2}{1+z}\right)\left[\frac{1}{4} \ln ^{3} z-\ln z \ln ^{2}(1-z)-2 \ln z \ln (1-z) \ln (1+z)\right.
\end{aligned}
$$




$$
\begin{aligned}
& +\frac{1}{2} \ln ^{2} z \ln (1-z)-2 \ln (1-z) \operatorname{Li}_{2}(1-z)-2 \ln (1+z) \operatorname{Li}_{2}(1-z)+\ln z \operatorname{Li}_{2}(1-z)-\ln z \operatorname{Li}_{2}(-z) \\
& \left.\left.\left.+2 \operatorname{Li}_{3}(1-z)+\mathrm{S}_{12}(1-z)+\mathrm{S}_{12}\left(z^{2}\right)\right]\right)\right)
\end{aligned}
$$

Here the factor $(-1)^{n}$ originates from the non-planar diagrams (namely, 13, 17, and 18 in Fig. 2 of [3]). It multiplies that part of the matrix element which is needed for the mass factorization of physical processes with two identical quarks in the final state. The unphysical part [see Eq. (2.19)] is equal to

$$
\begin{aligned}
& \hat{A}_{q q}^{\mathrm{NS}, \mathrm{EOM}}\left(z, \frac{-p^{2}}{\mu^{2}}, \frac{1}{\varepsilon}\right)=F C_{F}\{4 z-2(1-\hat{\xi})+\varepsilon[2 z-(1-\hat{\xi})][-1+\ln z+\ln (1-z)]\}+F^{2}\left(\frac { 1 } { \varepsilon } \left\{C_{F}^{2}\{-16-8 z\right.\right. \\
& \left.+16 z[-2 \ln (1-z)+\ln z]\}+C_{A} C_{F}\left[\frac{20}{3}-\frac{88}{3} z\right]+C_{F} T_{f}\left[-\frac{16}{3}+\frac{32}{3} z\right]\right\}+C_{F}^{2}\left[\frac{112}{3}-\frac{88}{3} z\right. \\
& +\zeta(2)\left(-8+16 z-\frac{32}{3} z^{2}\right)-12 z \ln (1-z)-24 z \ln ^{2}(1-z)-\frac{4}{3} \ln z(10-29 z)+8(1-4 z) \ln z \ln (1-z) \\
& +\frac{16}{3}\left(\frac{1}{z}-3 z-2 z^{2}\right)\left[\ln z \ln (1+z)+\operatorname{Li}_{2}(-z)\right]+\ln ^{2} z\left(12 z+\frac{16}{3} z^{2}\right)+16 \operatorname{Li}_{2}(1-z)(1-3 z) \\
& +8 z(1-z)\left\{-2 \ln (1-z) \zeta(2)+2 \ln z \zeta(2)-\ln ^{2} z \ln (1-z)-4 \ln z \operatorname{Li}_{2}(1-z)-2 \ln (1-z) \operatorname{Li}_{2}(1-z)\right. \\
& \left.\left.+6 \zeta(3)+6 \mathrm{Li}_{3}(1-z)-6 \mathrm{~S}_{12}(1-z)\right\}\right]+C_{A} C_{F}\left[-\frac{86}{9}+\frac{496}{9} z+4 \zeta(2)\left(1-2 z+\frac{4}{3} z^{2}\right)\right. \\
& -\frac{16}{3} \ln (1-z)(1+4 z)+\ln z(6-28 z)-\frac{8}{3} z^{2} \ln ^{2} z-4\left[\ln z \ln (1-z)+2 \operatorname{Li}_{2}(1-z)\right](1-2 z) \\
& -\frac{8}{3}\left(\frac{1}{z}-3 z-2 z^{2}\right)\left[\ln z \ln (1+z)+\mathrm{Li}_{2}(-z)\right]+8 z(1-z)\left\{-\zeta(2) \ln z+\frac{1}{2} \ln ^{2} z \ln (1-z)+\zeta(2) \ln (1\right. \\
& \left.\left.-z)+\ln (1-z) \mathrm{Li}_{2}(1-z)+2 \ln z \operatorname{Li}_{2}(1-z)-3 \zeta(3)-3 \operatorname{Li}_{3}(1-z)+3 \mathrm{~S}_{12}(1-z)\right\}\right]+n_{f} C_{F} T_{f}\left[\frac{8}{9}(5\right. \\
& \left.-16 z)-\frac{8}{3}(1-2 z)\{\ln z+2 \ln (1-z)\}\right]-\frac{16}{3}(-1)^{n}\left(C_{F}^{2}-\frac{1}{2} C_{A} C_{F}\right)\left\{1-z+z^{2}\left[-2 \zeta(2)+\ln ^{2} z\right]\right. \\
& \left.\left.-\ln z(1-2 z)+\left(\frac{1}{z}-3 z-2 z^{2}\right)\left[\ln z \ln (1+z)+\operatorname{Li}_{2}(-z)\right]\right\}\right) \text {. }
\end{aligned}
$$

Now we give the pure singlet terms [see Eq. (2.21)]

$$
\begin{aligned}
\hat{A}_{q q}^{\mathrm{PS}, \text { phys }}\left(z, \frac{-p^{2}}{\mu^{2}}, \frac{1}{\varepsilon}\right)= & F^{2} n_{f} C_{F} T_{f}\left(\frac{16}{\varepsilon^{2}}\{5(1-z)+2 \ln z(1+z)\}+\frac{8}{\varepsilon}\{(1-z)[7+10 \ln (1-z)]+\ln z(11+3 z)+4(1+z)\right. \\
& \left.\times\left[\ln z \ln (1-z)+\frac{3}{4} \ln ^{2} z+\mathrm{Li}_{2}(1-z)\right]\right\}+4(1-z)\left[10+5 \zeta(2)+14 \ln (1-z)+10 \ln ^{2}(1-z)\right] \\
& +20 \ln z(3+z)+8 \ln z \ln (1-z)(11+3 z)+2 \ln ^{2} z(23+19 z)+16 \operatorname{Li}_{2}(1-z)(3+4 z)+16(1+z) \\
& \times\left[2 \ln z \operatorname{Li}_{2}(1-z)+\frac{1}{2} \ln z \zeta(2)+\frac{3}{2} \ln ^{2} z \ln (1-z)+\frac{7}{12} \ln ^{3} z+\ln z \ln ^{2}(1-z)+2 \ln (1-z) \mathrm{Li}_{2}(1-z)\right. \\
& \left.\left.-2 \mathrm{Li}_{3}(1-z)+\mathrm{S}_{12}(1-z)\right]\right)
\end{aligned}
$$

The result for Eq. (2.22) is 


$$
\begin{aligned}
\hat{A}_{q q}^{\mathrm{PS}, \mathrm{EOM}}\left(z, \frac{-p^{2}}{\mu^{2}}, \frac{1}{\varepsilon}\right)= & 32 F^{2} n_{f} C_{F} T_{f}\left[\frac{1}{\varepsilon}\{-3(1-z)-\ln z(1+2 z)\}-3 \ln (1-z)(1-z)\right. \\
& \left.-\frac{1}{2} \ln z(5+z)-(1+2 z)\left(\ln z \ln (1-z)+\frac{3}{4} \ln ^{2} z+\mathrm{Li}_{2}(1-z)\right)\right] .
\end{aligned}
$$

The OME in Eq. (2.24) is equal to

$$
\begin{aligned}
& \hat{A}_{q g}^{\mathrm{phys}}\left(z, \frac{-p^{2}}{\mu^{2}}, \frac{1}{\varepsilon}\right)=F n_{f} T_{f}\left[-\frac{8}{\varepsilon}[1-2 z]+4-4(1-2 z)[\ln z+\ln (1-z)]+\varepsilon(2 \ln (1-z)\right. \\
& \left.\left.+2 \ln z-(1-2 z)\left[\zeta(2)+\{\ln z+\ln (1-z)\}^{2}\right]\right)\right]+F^{2} n_{f}\left(\frac { 8 } { \varepsilon ^ { 2 } } \left\{C _ { A } T _ { f } \left[\frac{2}{3}(25-14 z)-4 \ln (1-z)(1-2 z)\right.\right.\right. \\
& \left.+8 \ln z(1+z)]+C_{F} T_{f}[3+2(1-2 z)\{-2 \ln (1-z)+\ln z\}]+n_{f} T_{f}^{2} \frac{8}{3}(1-2 z)\right\} \\
& +\frac{1}{\varepsilon}\left\{C _ { A } T _ { f } \left[-\frac{8}{9}(44-z)-16 \zeta(2)(1-4 z)+64 \ln z \ln (1-z)(1+z)-24 \ln ^{2}(1-z)(1-2 z)\right.\right. \\
& +\frac{8}{3} \ln (1-z)(73-62 z)+\frac{16}{3} \ln z(14+11 z)+8 \ln ^{2} z(5+6 z)+96 \operatorname{Li}_{2}(1-z)+16(1+2 z) \\
& \left.\times\left\{\ln z \ln (1+z)+\mathrm{Li}_{2}(-z)\right\}\right]+C_{F} T_{f}[-4(12-13 z)+8 \ln (1-z)(3+4 z)-4 \ln z(5+8 z)+4(1-2 z) \\
& \left.\times\left\{4 \zeta(2)-6 \ln ^{2}(1-z)-8 \ln z \ln (1-z)+3 \ln ^{2} z-12 \operatorname{Li}_{2}(1-z)\right\}\right]+n_{f} T_{f}^{2}\left[-\frac{64}{9}(4-5 z)\right. \\
& \left.\left.+\frac{32}{3}(1-2 z)\{\ln z+\ln (1-z)\}\right]\right\}+C_{A} T_{f}\left[\frac{4}{27}(1348-1145 z)-8 \zeta(2)\left(-6+8 z+\frac{1}{3} z^{2}\right)\right. \\
& -8 \zeta(3)\left(2+z-6 z^{2}\right)-8 \ln (1-z) \zeta(2)\left(3-7 z+2 z^{2}\right)-\frac{28}{3} \ln ^{3}(1-z)(1-2 z)+\frac{2}{3} \ln ^{2}(1-z)(169-158 z) \\
& +8 \ln (1-z) \operatorname{Li}_{2}(1-z)\left(12+9 z-2 z^{2}\right)-\frac{4}{9} \ln (1-z)(206-145 z)+8 \ln z \zeta(2)\left(1+3 z+2 z^{2}\right) \\
& +8 \ln z \ln ^{2}(1-z)(5+6 z)+\frac{4}{3} \ln z \ln (1-z)(73+10 z)+4 \ln ^{2} z \ln (1-z)\left(11+11 z-2 z^{2}\right) \\
& +\frac{4}{3} \ln ^{3} z(11+14 z)+\frac{4}{3} \ln ^{2} z\left(26+47 z+z^{2}\right)+16 \ln z \operatorname{Li}_{2}(1-z)\left(4+3 z-2 z^{2}\right)+\frac{16}{9} \ln z(31+58 z) \\
& -8 \mathrm{Li}_{2}(1-z)(1-12 z)-8\left(3-\frac{2}{3 z}+4 z+\frac{1}{3} z^{2}\right)\left\{\ln z \ln (1+z)+\operatorname{Li}_{2}(-z)\right\}-8 \mathrm{Li}_{3}(1-z)\left(8+19 z-6 z^{2}\right) \\
& -8 \mathrm{~S}_{12}(1-z)\left(1-13 z+6 z^{2}\right)+8(1+2 z)\left\{2 \ln (1+z) \zeta(2)+2 \ln (1+z) \operatorname{Li}_{2}(1-z)+4 \ln (1+z) \mathrm{Li}_{2}(-z)\right. \\
& +2 \ln z \ln (1-z) \ln (1+z)+2 \ln z \ln ^{2}(1+z)+\frac{1}{2} \ln ^{2} z \ln (1+z)+\ln z \mathrm{Li}_{2}(-z)-\mathrm{Li}_{3}(-z)+6 \mathrm{~S}_{12}(-z) \\
& \left.\left.-\mathrm{S}_{12}\left(z^{2}\right)\right\}\right]+C_{F} T_{f}\left[2(23-11 z)+2 \zeta(2)(3-8 z)+12 \ln ^{2}(1-z)(1+2 z)-32 \ln (1-z)(2-3 z)\right. \\
& -4 \ln z \ln (1-z)(5-4 z)-3 \ln ^{2} z(7+8 z)+2 \ln z(2-13 z)-32 \operatorname{Li}_{2}(1-z)(1-z)
\end{aligned}
$$




$$
\begin{aligned}
& +4(1-2 z)\left\{10 \zeta(3)+2 \ln (1-z) \zeta(2)-\frac{7}{3} \ln ^{3}(1-z)-10 \ln (1-z) \operatorname{Li}_{2}(1-z)+5 \ln z \zeta(2)\right. \\
& \left.\left.-7 \ln z \ln ^{2}(1-z)-5 \ln ^{2} z \ln (1-z)+\frac{7}{6} \ln ^{3} z-16 \ln z \operatorname{Li}_{2}(1-z)+6 \operatorname{Li}_{3}(1-z)-12 \mathrm{~S}_{12}(1-z)\right\}\right] \\
& \left.+n_{f} T_{f}^{2} \frac{8}{3}\left[\frac{86}{9}-\frac{112}{9} z-\frac{8}{3}(4-5 z)\{\ln z+\ln (1-z)\}+(1-2 z)\{\ln z+\ln (1-z)\}^{2}\right]\right)
\end{aligned}
$$

The next OME $\hat{A}_{g q}$ can be split into physical and unphysical parts according to Eq. (2.25). The former becomes [see Eq. (2.26)]

$$
\begin{aligned}
& \hat{A}_{g q}^{\text {phys }}\left(z, \frac{-p^{2}}{\mu^{2}}, \frac{1}{\varepsilon}\right)=F C_{F}\left[\frac{1}{\varepsilon}[8-4 z]+(4-2 z)[\ln z+\ln (1-z)]+2+\varepsilon\left\{\left(1-\frac{z}{2}\right)\left[\zeta(2)+\{\ln (1-z)+\ln z\}^{2}\right]+\ln (1-z)\right.\right. \\
& +\ln z\}]+F^{2}\left(\frac { 1 } { \varepsilon ^ { 2 } } \left\{C_{F}^{2}[12 z+8(2-z)\{2 \ln (1-z)-\ln z\}]+C_{A} C_{F}\left[-\frac{8}{3}(14-25 z)+16 \ln (1-z)(2-z)\right.\right.\right. \\
& \left.-16 \ln z(4+z)]-n_{f} C_{F} T_{f} \frac{32}{3}(2-z)\right\}+\frac{1}{\varepsilon}\left\{C_{F}^{2}[-14+4 z-4 \ln (1-z)(4-7 z)-2 z \ln z\right. \\
& \left.-6(2-z)\left(-\frac{8}{3} \zeta(2)-2 \ln ^{2}(1-z)+\frac{4}{3} \ln z \ln (1-z)+\ln ^{2} z+4 \operatorname{Li}_{2}(1-z)\right)\right] \\
& +C_{A} C_{F}\left[-\frac{4}{9}(47-37 z)-8 \zeta(2)(4-z)+12 \ln ^{2}(1-z)(2-z)-\frac{4}{3} \ln (1-z)(26-49 z)-8 \ln z \ln (1-z)\right. \\
& \left.\times(2+5 z)-4 \ln ^{2} z(10+3 z)-\frac{4}{3} \ln z(40+7 z)-48 z \operatorname{Li}_{2}(1-z)-8(2+z)\left\{\ln z \ln (1+z)+\operatorname{Li}_{2}(-z)\right\}\right] \\
& \left.-n_{f} C_{F} T_{f} \frac{16}{3}(2-z)\left[\frac{1}{3}+\ln (1-z)+2 \ln z\right]\right\}+C_{F}^{2}[25-11 z-3 \zeta(2)(4-3 z)-16 \zeta(3)(1-2 z) \\
& -2 \ln (1-z)(4-z)-6 \ln ^{2}(1-z)(2-3 z)-8 \ln (1-z) \operatorname{Li}_{2}(1-z)(4-z)-2 \ln z \zeta(2)(2-5 z) \\
& -4 \ln z \ln (1-z)(1-2 z)+4 \ln (1-z) \zeta(2)(10-7 z)-2 \ln ^{2} z \ln (1-z)(6-z) \\
& -\frac{7}{3}(2-z)\left\{-2 \ln ^{3}(1-z)+\frac{12}{7} \ln z \ln ^{2}(1-z)+\ln ^{3} z-\frac{12}{7} \operatorname{Li}_{2}(1-z)\right\}-\frac{9}{2} z \ln ^{2} z-8 \ln z \operatorname{Li}_{2}(1-z)(2+z) \\
& \left.-3 \ln z(1+2 z)+24 z \mathrm{Li}_{3}(1-z)+12 \mathrm{~S}_{12}(1-z)(2-3 z)\right]+C_{A} C_{F}\left[-\frac{2}{27}(1400-1573 z)\right. \\
& -\frac{2}{3} \zeta(2)\left(2-25 z+14 z^{2}\right)+4 \zeta(3)\left(1-10 z+6 z^{2}\right)-4 \ln (1-z) \zeta(2)\left(7-7 z+2 z^{2}\right)+\frac{14}{3} \ln ^{3}(1-z)(2-z) \\
& -\frac{1}{3} \ln ^{2}(1-z)(50-97 z)-2\left(7+9 z+2 z^{2}\right)\left\{\ln ^{2} z \ln (1-z)+2 \ln (1-z) \operatorname{Li}_{2}(1-z)\right\}+\frac{8}{9} \ln (1-z)(13-2 z) \\
& -4 \ln z \zeta(2)\left(5+4 z-2 z^{2}\right)-2 \ln z \ln ^{2}(1-z)(2+15 z)-\frac{4}{3} \ln z \ln (1-z)(35+8 z)-\frac{2}{3} \ln ^{3} z(22+7 z) \\
& -\frac{1}{3} \ln ^{2} z\left(110+71 z-14 z^{2}\right)-\frac{2}{9} \ln z(421+145 z)-8 \ln z \operatorname{Li}_{2}(1-z)\left(-1+2 z+2 z^{2}\right) \\
& -4 \mathrm{Li}_{2}(1-z)(6+11 z)-\frac{4}{3}\left(-9+\frac{1}{z}-3 z+7 z^{2}\right)\left\{\ln z \ln (1+z)+\operatorname{Li}_{2}(-z)\right\}+4 \mathrm{Li}_{3}(1-z)\left(13-z+6 z^{2}\right) \\
& +4 \mathrm{~S}_{12}(1-z)\left(5+4 z-6 z^{2}\right)+4(2+z)\left\{-2 \ln (1+z) \zeta(2)-2 \ln (1+z) \operatorname{Li}_{2}(1-z)-4 \ln (1+z) \operatorname{Li}_{2}(-z)\right. \\
& \text { 076002-12 }
\end{aligned}
$$




$$
\begin{aligned}
& -2 \ln z \ln (1-z) \ln (1+z)-2 \ln z \ln ^{2}(1+z)-\frac{1}{2} \ln ^{2} z \ln (1+z)-\ln z \operatorname{Li}_{2}(-z)+\operatorname{Li}_{3}(-z)-6 \mathrm{~S}_{12}(-z) \\
& \left.\left.\left.+\mathrm{S}_{12}\left(z^{2}\right)\right\}\right]-\frac{4}{3} n_{f} C_{F} T_{f}(2-z)\left[\frac{32}{9}+\frac{2}{3} \ln (1-z)+\frac{4}{3} \ln z+2 \zeta(2)+\{\ln (1-z)+2 \ln z\}^{2}\right]\right)
\end{aligned}
$$

The unphysical part given by Eq. (2.27) is equal to

$$
\begin{aligned}
\hat{A}_{g q}^{\mathrm{EOM}}\left(z, \frac{-p^{2}}{\mu^{2}}, \frac{1}{\varepsilon}\right)= & F C_{F}\{-4+4 z-2 \varepsilon(1-z)[\ln z+\ln (1-z)]\}+F^{2}\left(\frac { 1 6 } { \varepsilon } \left\{C_{F}^{2}[2-2 z+z \ln z]+n_{f} C_{F} T_{f} \frac{4}{3}(1-z)\right.\right. \\
& \left.+C_{A} C_{F}\left(\frac{13}{3}(1-z)-2 \ln (1-z)(1-z)+2 \ln z(2+z)\right)\right\}+C_{F}^{2}[-40(1-z)-16(1-z) \\
& \left.\times\left\{\zeta(2)-\frac{9}{4} \ln (1-z)\right\}+16 \ln z \ln (1-z)+12 z \ln ^{2} z+16 \ln z(1-2 z)+16 \operatorname{Li}_{2}(1-z)(2-z)\right] \\
& +C_{A} C_{F}\left[-\frac{148}{9}(1-z)+\frac{4}{3} \zeta(2)\left(15-6 z+7 z^{2}\right)-24 \ln ^{2}(1-z)(1-z)+\frac{224}{3} \ln (1-z)(1-z)\right. \\
& +24 \ln z \ln (1-z)(1+3 z)+16 \operatorname{Li}_{2}(1-z)(1+5 z)+\frac{4}{3} \ln z(33+z)+\frac{4}{3}\left(9+\frac{4}{z}+12 z+7 z^{2}\right) \\
& \times\left\{\ln z \ln (1+z)+\mathrm{Li}_{2}(-z)\right\}+\frac{2}{3} \ln ^{2} z\left(63+36 z-7 z^{2}\right)+4\left(1-3 z+2 z^{2}\right)\left\{\ln (1-z) \mathrm{Li}_{2}(1-z)\right. \\
& \left.\left.-\ln z \zeta(2)+\ln (1-z) \zeta(2)+\frac{1}{2} \ln ^{2} z \ln (1-z)+2 \ln z \operatorname{Li}_{2}(1-z)-3 \zeta(3)-3 \operatorname{Li}_{3}(1-z)+3 \mathrm{~S}_{12}(1-z)\right\}\right] \\
& \left.+n_{f} C_{F} T_{f} \frac{32}{3}(1-z)\left[-\frac{2}{3}+\ln (1-z)+2 \ln z\right]\right) .
\end{aligned}
$$

The gluonic OME in Eq. (2.29) equals

$$
\begin{aligned}
& \hat{A}_{g g}^{\text {phys }}\left(z, \frac{-p^{2}}{\mu^{2}}, \frac{1}{\varepsilon}\right)=F\left(\frac{1}{\varepsilon}\left\{C_{A}\left[8-16 z+\frac{8}{1-z}+\frac{22}{3} \delta(1-z)\right]-\frac{8}{3} n_{f} T_{f} \delta(1-z)\right\}+C_{A}\left[-2+(1-\hat{\xi}) \frac{1}{1-z}+\left(4-8 z+\frac{4}{1-z}\right)\right.\right. \\
& \left.\times\{\ln z+\ln (1-z)\}+\delta(1-z)\left\{-\frac{67}{9}+4 \zeta(2)-(1-\hat{\xi})+\frac{1}{4}(1-\hat{\xi})^{2}\right\}\right]+n_{f} T_{f} \frac{20}{9} \delta(1-z) \\
& +\varepsilon\left\{C _ { A } \left[\left(1-2 z+\frac{1}{1-z}\right)\left\{\zeta(2)+[\ln z+\ln (1-z)]^{2}\right\}-\ln (1-z)-\ln z+\frac{1}{2} \frac{1-\hat{\xi}}{1-z}\{-1+\ln z+\ln (1-z)\}\right.\right. \\
& \left.\left.\left.+\delta(1-z)\left(\frac{202}{27}-\frac{11}{12} \zeta(2)-\frac{14}{3} \zeta(3)+\frac{1}{4} \zeta(2)(1-\hat{\xi})-\frac{1}{4}(1-\hat{\xi})^{2}\right)\right]+n_{f} T_{f} \delta(1-z)\left(-\frac{56}{27}+\frac{1}{3} \zeta(2)\right)\right\}\right) \\
& +F^{2}\left(\frac { 1 } { \varepsilon ^ { 2 } } \left\{C _ { A } ^ { 2 } \left[-168+80 z+\frac{88}{1-z}+4 \delta(1-z)\left(\frac{121}{9}-8 \zeta(2)\right)+64 \ln (1-z)\left(1-2 z+\frac{1}{1-z}\right)\right.\right.\right. \\
& \left.-32 \ln z\left(3+\frac{1}{1-z}\right)\right]-n_{f} C_{A} T_{f} 32\left[1-2 z+\frac{1}{1-z}+\frac{11}{9} \delta(1-z)\right]+n_{f} C_{F} T_{f}[80(1-z)+32 \ln z(1+z)] \\
& \left.+n_{f}^{2} T_{f}^{2} \frac{64}{9} \delta(1-z)\right\}+\frac{1}{\varepsilon}\left\{C _ { A } ^ { 2 } \left[\frac{2}{3}(119-29 z)-\frac{86}{1-z}+\delta(1-z)\left(-\frac{3326}{27}+\frac{176}{3} \zeta(2)+20 \zeta(3)\right)\right.\right. \\
& -8 \zeta(2)\left(4 z+\frac{1}{1+z}-\frac{1}{1-z}\right)+48 \ln ^{2}(1-z)\left(1-2 z+\frac{1}{1-z}\right)+\frac{16}{3} \ln (1-z)\left(-40+26 z+\frac{11}{1-z}\right)
\end{aligned}
$$




$$
\begin{aligned}
& -16 \ln z \ln (1-z)\left(3+6 z-\frac{1}{1-z}\right)-4 \ln ^{2} z\left(16-\frac{1}{1+z}+\frac{5}{1-z}\right)-\frac{4}{3} \ln z\left(57+21 z-\frac{44}{1-z}\right) \\
& \left.-64 \mathrm{Li}_{2}(1-z)(1+z)-16\left(1+2 z+\frac{1}{1+z}\right)\left\{\ln z \ln (1+z)+\mathrm{Li}_{2}(-z)\right\}\right]+n_{f} C_{A} T_{f}\left[\frac{16}{3}(10-13 z)+\frac{24}{1-z}\right. \\
& \left.+\frac{8}{3} \delta(1-z)\left(\frac{271}{9}-8 \zeta(2)\right)-\frac{64}{3} \ln (1-z)\left(1-2 z+\frac{1}{1-z}\right)-16 \ln z\left(1-3 z+\frac{4}{3} \frac{1}{1-z}\right)\right]+n_{f} C_{F} T_{f} \\
& {\left[-88(1-z)+4 \delta(1-z)+80 \ln (1-z)(1-z)+8 \ln z(3-7 z)+8(1+z)\left\{4 \ln z \ln (1-z)+3 \ln ^{2} z\right.\right.} \\
& \left.\left.\left.+4 \mathrm{Li}_{2}(1-z)\right\}\right]-n_{f}^{2} T_{f}^{2} \frac{320}{27} \delta(1-z)\right\} \\
& +C_{A}^{2}\left[-\frac{4}{9}(565-403 z)+\frac{254}{3} \frac{1}{1-z}-\zeta(2)\left(\frac{149}{3}-\frac{181}{3} z+8 z^{2}-\frac{19}{1-z}\right)+\delta(1-z)\left(\frac{11141}{54}-\frac{214}{3} \zeta(2)\right.\right. \\
& \left.-\frac{854}{9} \zeta(3)+5 \zeta(2)^{2}\right)+\zeta(3)\left(-14+52 z+15 z^{2}-\frac{4}{1+z}-\frac{10}{1-z}\right)+\ln (1-z) \zeta(2)\left(10-28 z-5 z^{2}\right. \\
& \left.+\frac{18}{1-z}\right)+\frac{56}{3} \ln ^{3}(1-z)\left(1-2 z+\frac{1}{1-z}\right)+2 \ln ^{2}(1-z)\left(-59+42 z+\frac{11}{1-z}\right) \\
& -\ln (1-z) \operatorname{Li}_{2}(1-z)\left(74+108 z+5 z^{2}+\frac{16}{1+z}-\frac{2}{1-z}\right)-\frac{1}{9} \ln (1-z)\left(-1267+959 z+\frac{506}{1-z}\right) \\
& -4\left(5+8 z+\frac{2}{1+z}\right)\left\{\ln (1+z) \zeta(2)+2 \ln (1+z) \operatorname{Li}_{2}(-z)\right\}-\ln z \zeta(2)\left(30-12 z-5 z^{2}+\frac{10}{1-z}\right) \\
& -4 \ln z \ln ^{2}(1-z)\left(5+22 z+\frac{2}{1+z}-\frac{5}{1-z}\right)+\frac{1}{3} \ln z \ln (1-z)\left(-245-29 z+\frac{97}{1-z}\right) \\
& -4 \ln z \ln ^{2}(1+z)\left(5+8 z+\frac{2}{1+z}\right)-2 \ln ^{2} z \ln (1+z)\left(1+4 z+\frac{4}{1+z}\right)-\ln ^{2} z \ln (1-z)\left(37+54 z+\frac{5}{2} z^{2}\right. \\
& \left.-\frac{4}{1+z}-\frac{5}{1-z}\right)-2 \ln ^{3} z\left(12-\frac{1}{1+z}+\frac{11}{3} \frac{1}{1-z}\right) \\
& -\ln ^{2} z\left(31+41 z-4 z^{2}-\frac{22}{1-z}\right)-2 \ln z \operatorname{Li}_{2}(1-z)\left(10+60 z+5 z^{2}-\frac{4}{1+z}-\frac{14}{1-z}\right) \\
& -4 \ln z \operatorname{Li}_{2}(-z)\left(1+4 z+\frac{2}{1+z}+\frac{2}{1-z}\right)-\ln z\left(\frac{226}{3}+74 z+\frac{521}{9} \frac{1}{1-z}\right)-2 \operatorname{Li}_{2}(1-z) \\
& \times\left(-21+45 z+\frac{13}{3} \frac{1}{1-z}\right)+4\left(8-\frac{1}{z}+7 z-2 z^{2}\right) \\
& \times\left\{\ln z \ln (1+z)+\mathrm{Li}_{2}(-z)\right\}+\mathrm{Li}_{3}(1-z)\left(62+132 z+15 z^{2}+\frac{16}{1+z}-\frac{6}{1-z}\right)+4 \mathrm{Li}_{3}(-z) \\
& \times\left(1+4 z+\frac{4}{1-z}\right)+S_{12}(1-z)\left(46-148 z-15 z^{2}+\frac{8}{1+z}+\frac{46}{1-z}\right)-8 S_{12}(-z)\left(7+12 z+\frac{4}{1+z}\right) \\
& \left.+8\left(1+2 z+\frac{1}{1+z}\right)\left\{-2 \ln (1+z) \operatorname{Li}_{2}(1-z)-2 \ln z \ln (1-z) \ln (1+z)+\mathrm{S}_{12}\left(z^{2}\right)\right\}\right] \\
& +n_{f} C_{A} T_{f}\left[-\frac{2}{9}(473-563 z)-\frac{64}{3} \frac{1}{1-z}+\frac{4}{3} \zeta(2)\left(13+10 z+4 z^{2}-\frac{6}{1-z}\right)+\delta(1-z)\left(-\frac{3224}{27}\right.\right. \\
& \left.+\frac{236}{9} \zeta(2)+\frac{136}{9} \zeta(3)\right)-8 \ln ^{2}(1-z)\left(1-2 z+\frac{1}{1-z}\right)+\frac{4}{9} \ln (1-z)\left(97-131 z+\frac{34}{1-z}\right)
\end{aligned}
$$




$$
\begin{aligned}
& -\frac{4}{3} \ln z \ln (1-z)\left(11-22 z+\frac{8}{1-z}\right)-4 \ln ^{2} z\left(3-5 z+\frac{2}{3} z^{2}+\frac{2}{1-z}\right)+\frac{4}{3} \ln z\left(10-37 z+\frac{37}{3} \frac{1}{1-z}\right) \\
& -\frac{8}{3} \operatorname{Li}_{2}(1-z)\left(3-\frac{2}{1-z}\right)+\frac{16}{3}\left(3+\frac{1}{z}+3 z+z^{2}\right)\left\{\ln z \ln (1+z)+\mathrm{Li}_{2}(-z)\right\} \\
& +8 z^{2}\left(\ln (1-z) \mathrm{Li}_{2}(1-z)-\ln z \zeta(2)+\frac{1}{2} \ln ^{2} z \ln (1-z)+\ln (1-z) \zeta(2)+2 \ln z \operatorname{Li}_{2}(1-z)\right. \\
& \left.\left.-3 \zeta(3)-3 \mathrm{Li}_{3}(1-z)+3 \mathrm{~S}_{12}(1-z)\right)\right]+n_{f} C_{F} T_{f}\left[\delta(1-z)\left(-\frac{55}{3}+16 \zeta(3)\right)+\frac{568}{3}(1-z)\right. \\
& -4 \zeta(2)\left(3+5 z+\frac{8}{3} z^{2}\right)+40 \ln ^{2}(1-z)(1-z)-88 \ln (1-z)(1-z)+8 \ln z \ln (1-z)(3-7 z) \\
& +2 \ln ^{2} z\left(7-11 z+\frac{8}{3} z^{2}\right)-\frac{4}{3} \ln z(1-65 z) \\
& -\frac{32}{3}\left(3+\frac{1}{z}+3 z+z^{2}\right)\left\{\ln z \ln (1+z)+\mathrm{Li}_{2}(-z)\right\}+16(1+z)\left(2 \ln (1-z) \mathrm{Li}_{2}(1-z)+\frac{1}{2} \ln z \zeta(2)\right. \\
& +\ln z \ln ^{2}(1-z)+\frac{3}{2} \ln ^{2} z \ln (1-z)+\frac{7}{12} \ln ^{3} z+2 \ln z \mathrm{Li}_{2}(1-z)-\mathrm{Li}_{2}(1-z) \\
& \left.\left.-2 \mathrm{Li}_{3}(1-z)+\mathrm{S}_{12}(1-z)\right)\right]+n_{f}^{2} T_{f}^{2} 16 \delta(1-z)\left[1-\frac{1}{9} \zeta(2)\right] \text {. }
\end{aligned}
$$

Finally we have determined the unrenormalized $\hat{Z}_{q q}^{5, \mathrm{NS}}$ from Eq. (2.15). The numerator, which is equal to the spin averaged OME $\hat{A}_{q q}^{\mathrm{NS} \text {,phys }}$, is given in Eq. (A3) of Ref. [12]. The denominator has been computed above [see Eq. (A3)]. The result is equal to

$$
\begin{aligned}
\hat{Z}_{q q}^{5, \mathrm{NS}}= & \delta(1-z)+\hat{a}_{s} S_{\varepsilon} C_{F}(-8(1-z)+\varepsilon\{-4(1-z)[\ln (1-z)+\ln z]+2 z-(1-\hat{\xi})\})+\hat{a}_{s}^{2} S_{\varepsilon}^{2}\left[C_{F}^{2}\{-16(1-z)-8(2+z) \ln z\right. \\
& +16(1-z) \ln z \ln (1-z)\}+C_{A} C_{F}\left\{\frac{1}{\varepsilon} \frac{88}{3}(1-z)-\frac{562}{9}+\frac{460}{9} z+8(1-z) \zeta(2)+\frac{88}{3}(1-z) \ln (1-z)+\left(\frac{8}{3}-\frac{80}{3} z\right) \ln z\right. \\
& \left.-4(1-z) \ln ^{2} z\right\}+n_{f} C_{F} T_{f}\left\{-\frac{1}{\varepsilon} \frac{32}{3}(1-z)+\frac{56}{9}-\frac{32}{9} z-\frac{32}{3}(1-z) \ln (1-z)-\frac{16}{3}(1-z) \ln z\right\}+(-1)^{n}\left(C_{F}^{2}-\frac{1}{2} C_{A} C_{F}\right) \\
& \left.\times\left\{8(1+z)\left[4 \operatorname{Li}_{2}(-z)+4 \ln z \ln (1+z)+2 \zeta(2)-\ln ^{2} z-3 \ln z\right]-56(1-z)\right\}\right]
\end{aligned}
$$

[1] Proceedings of the 1995/96 workshop Future Physics at HERA, edited by G. Ingelman, A. de Roeck, and R. Klanner, DESY, Hamburg, 1996 (unpublished), Vol. 2, pp. 757-882.

[2] Proceedings of the 2nd Topical Workshop Deep Inelastic Scattering off Polarized Targets: Theory Meets Experiment, DESY, Hamburg, 1997, edited by J. Bluemlein, A. de Roeck, T. Gehrmann, and W. Nowak (unpublished).

[3] R. Mertig and W. L. van Neerven, Z. Phys. C 70, 637 (1996).

[4] W. Vogelsang, Phys. Rev. D 54, 2023 (1996); Nucl. Phys. B475, 47 (1996).

[5] J. Kodaira, S. Matsuda, T. Muta, T. Kematsu, and K. Sasaki, Phys. Rev. D 20, 627 (1979).
[6] G. T. Bodwin and J. Qiu, Phys. Rev. D 41, 2755 (1979).

[7] E. B. Zijlstra and W. L. van Neerven, Nucl. Phys. B417, 61 (1994); B426, 245(E) (1994).

[8] G. 't Hooft and M. Veltman, Nucl. Phys. B44, 189 (1972).

[9] F. Breitenlohner and D. Maison, Commun. Math. Phys. 52, 11 (1977); 52, 39 (1977); 52, 55 (1977).

[10] C. Becchi, A. Rouet, and R. Stora, Phys. Lett. 52B, 344 (1974); Commun. Math. Phys. 42, 127 (1975); Ann. Phys. (N.Y.) 98, 287 (1976).

[11] K. Sasaki, Prog. Theor. Phys. 54, 1816 (1975); M. A. Ahmed and G. G. Ross, Nucl. Phys. B111, 441 (1976). 
[12] Y. Matiounine, J. Smith, and W. L. van Neerven, Phys. Rev. D 57, 6701 (1998).

[13] J. A. Dixon and J. C. Taylor, Nucl. Phys. B78, 552 (1974).

[14] H. Kluberg-Stern and J. B. Zuber, Phys. Rev. D 12, 467 (1975); 12, 3159 (1975).

[15] S. D. Joglekar and B. W. Lee, Ann. Phys. (N.Y.) 97, 160 (1976); S. D. Joglekar, ibid. 108, 233 (1977); 109, 210 (1977).

[16] R. Hamberg and W. L. van Neerven, Nucl. Phys. B379, 143 (1992); R. Hamberg, Ph.D. thesis, University of Leiden, 1991.

[17] J. C. Collins and R. J. Scalise, Phys. Rev. D 50, 4117 (1994).

[18] B. W. Harris and J. Smith, Phys. Rev. D 51, 4550 (1995).

[19] M. Buza, Y. Matiounine, J. Smith, R. Migneron, and W. L. van Neerven, Nucl. Phys. B472, 611 (1996).

[20] J. A. M. Vermaseren, FORM2, published by Computer Algebra Netherlands (CAN), Kruislaan 413, 1098SJ Amsterdam, The Netherlands.

[21] D. Akyeamopong and R. Delbourgo, Nuovo Cimento A 17,
578 (1973); 18, 94 (1973); 19, 219 (1974).

[22] S. A. Larin, Phys. Lett. B 303, 113 (1993); 334, 192 (1994).

[23] S. A. Larin and J. A. M. Vermaseren, Phys. Lett. B 259, 345 (1991).

[24] S. L. Adler and W. Bardeen, Phys. Rev. 182, 1517 (1969).

[25] P. J. Rijken and W. L. van Neerven, Nucl. Phys. B523, 245 (1998).

[26] V. N. Gribov and L. N. Lipatov, Sov. J. Nucl. Phys. 15, 438 (1972); 15, 675 (1972).

[27] G. Curci, W. Furmanski, and R. Petronzio, Nucl. Phys. B175, 27 (1980).

[28] M. Stratmann and W. Vogelsang, Nucl. Phys. B496, 41 (1997).

[29] L. Lewin, Polylogarithms and Associated Functions (North Holland, Amsterdam, 1983); R. Barbieri, J. A. Mignaco, and E. Remiddi, Nuovo Cimento A 11, 824 (1972); A. Devoto and D. W. Duke, Riv. Nuovo Cimento 7, 1 (1984). 